\title{
Fine-mapping of 150 breast cancer risk regions identifies 178 high confidence target genes
}

Laura Fachal $^{1}$, Hugues Aschard ${ }^{2-4,275}$, Jonathan Beesley ${ }^{5,275}$, Daniel R. Barnes ${ }^{6}$, Jamie Allen ${ }^{6}$, Siddhartha Kar $^{1}$, Karen A. Pooley ${ }^{6}$, Joe Dennis ${ }^{6}$, Kyriaki Michailidou ${ }^{6,7}$, Constance Turman ${ }^{4}$, Penny Soucy ${ }^{8}$, Audrey Lemaçon ${ }^{8}$, Michael Lush $^{6}$, Jonathan P. Tyrer ${ }^{1}$, Maya Ghoussaini $^{1}$, Mahdi Moradi Marjaneh $^{5}$, Xia Jiang ${ }^{3}$, Simona Agata ${ }^{9}$, Kristiina Aittomäki ${ }^{10}$, M. Rosario Alonso ${ }^{11}$, Irene L. Andrulis ${ }^{12,13}$, Hoda Anton-Culver ${ }^{14}$, Natalia N. Antonenkova ${ }^{15}$, Adalgeir Arason ${ }^{16,17}$, Volker Arndt ${ }^{18}$, Kristan J. Aronson ${ }^{19}$, Banu K. Arun ${ }^{20}$, Bernd Auber ${ }^{21}$, Paul L. Auer22, 23, Jacopo Azzollini24, Judith Balmaña ${ }^{25}$, Rosa B. Barkardottir ${ }^{16,17}$, Daniel Barrowdale $^{6}$, Alicia Beeghly-Fadiel ${ }^{26}$, Javier Benitez ${ }^{27,28}$, Marina Bermisheva ${ }^{29}$, Katarzyna Białkowska ${ }^{30}$, Amie M. Blanco ${ }^{31}$, Carl Blomqvist ${ }^{32,33}$, William Blot ${ }^{26,34}$, Natalia V. Bogdanova ${ }^{15,35,36}$, Stig E. Bojesen ${ }^{37-}$ 39, Manjeet K. Bolla ${ }^{6}$, Bernardo Bonanni ${ }^{40}$, Ake Borg ${ }^{41}$, Kristin Bosse ${ }^{42}$, Hiltrud Brauch ${ }^{43-45}$, Hermann Brenner ${ }^{18,45,46}$, Ignacio Briceno ${ }^{47}$ 48, Ian W. Brock ${ }^{49}$, Angela Brooks-Wilson ${ }^{50,51}$, Thomas Brüning 52 , Barbara Burwinkel ${ }^{53,54}$, Saundra S. Buys ${ }^{55}$, Qiuyin Cai ${ }^{26}$, Trinidad Caldés ${ }^{56}$, Maria A. Caligo ${ }^{57}$, Nicola J. Camp $^{58}$, Ian Campbell ${ }^{59,60}$, Federico Canzian ${ }^{61}$, Jason S. Carroll ${ }^{62}$, Brian D. Carter ${ }^{63}$, Jose E. Castelao ${ }^{64}$, Jocelyne Chiquette ${ }^{65}$, Hans Christiansen ${ }^{35}$, Wendy K. Chung ${ }^{66}$, Kathleen B.M. Claes ${ }^{67}$, Christine L. Clarke $^{68}$, GEMO Study Collaborators ${ }^{69-71}$, EMBRACE Collaborators ${ }^{6}$, J. Margriet Collée ${ }^{72}$, Sten Cornelissen $^{73}$, Fergus J. Couch ${ }^{74}$, Angela Cox ${ }^{49}$, Simon S. Cross ${ }^{75}$, Cezary Cybulski ${ }^{30}$, Kamila Czene ${ }^{76}$, Mary B. Daly ${ }^{77}$, Miguel de la Hoya ${ }^{56}$, Peter Devilee ${ }^{78,79}$, Orland Diez ${ }^{25,80}$, Yuan Chun Ding ${ }^{81}$, Gillian S. Dite ${ }^{82}$, Susan M. Domchek ${ }^{83}$, Thilo Dörk ${ }^{36}$, Isabel dos-Santos-Silva ${ }^{84}$, Arnaud Droit ${ }^{8,}{ }^{85}$, Stéphane Dubois ${ }^{8}$, Martine Dumont ${ }^{86}$, Mercedes Duran ${ }^{87}$, Lorraine Durcan ${ }^{88,}{ }^{89}$, Miriam Dwek ${ }^{90}$, Diana M. Eccles ${ }^{89}$, Christoph Engel ${ }^{91,} 92$, Mikael Eriksson ${ }^{76}$, D. Gareth Evans ${ }^{93,}{ }^{94}$, Peter A. Fasching ${ }^{95,}$, , Olivia Fletcher $^{97}$, Giuseppe Floris $^{98}$, Henrik Flyger ${ }^{99}$, Lenka Foretova ${ }^{100}$, William D. Foulkes ${ }^{101}$, Eitan Friedman ${ }^{102,}$ 103, Lin 
Fritschi $^{104}$, Debra Frost ${ }^{6}$, Marike Gabrielson ${ }^{76}$, Manuela Gago-Dominguez ${ }^{105,}{ }^{106}$, Gaetana Gambino ${ }^{107}$, Patricia A. Ganz ${ }^{108}$, Susan M. Gapstur ${ }^{63}$, Judy Garber ${ }^{109}$, José A. García-Sáenz ${ }^{56}$, Mia M. Gaudet ${ }^{63}$, Vassilios Georgoulias ${ }^{110}$, Graham G. Giles ${ }^{82,111,112}$, Gord Glendon ${ }^{12}$, Andrew K. Godwin ${ }^{113}$, Mark S. Goldberg$^{114}$, 115, David E. Goldgar ${ }^{116}$, Anna González-Neira ${ }^{28}$, Mark H. Greene ${ }^{117}$, Mervi Grip ${ }^{118}$, Jacek Gronwald $^{30}$, Anne Grundy ${ }^{119}$, Pascal Guénel ${ }^{120}$, Eric Hahnen ${ }^{121}{ }^{122}$, Christopher A. Haiman ${ }^{123}$, Niclas Håkansson ${ }^{124}$, Per Hall ${ }^{76,125}$, Ute Hamann ${ }^{126}$, Patricia A. Harrington ${ }^{1}$, Jaana M. Hartikainen ${ }^{127-129}$, Mikael Hartman $^{130,}{ }^{131}$, Wei He ${ }^{76}$, Catherine S. Healey ${ }^{1}$, Bernadette A.M. Heemskerk-Gerritsen ${ }^{132}$, Jane Heyworth $^{133}$, Peter Hillemanns ${ }^{36}$, Frans B.L. Hogervorst ${ }^{134}$, Antoinette Hollestelle ${ }^{132}$, Maartje J. Hooning ${ }^{132}$, John L. Hopper ${ }^{82}$, Anthony Howell ${ }^{135}$, Guanmengqian Huang ${ }^{126}$, Peter J. Hulick ${ }^{136,137}$, Evgeny N. Imyanitov ${ }^{138}$, ABCTB Investigators ${ }^{139}$, KConFab Investigators ${ }^{59,60}$, HEBON Investigators ${ }^{140}$, Claudine Isaacs $^{141}$, Motoki Iwasaki ${ }^{142}$, Agnes Jager ${ }^{132}$, Milena Jakimovska ${ }^{143}$, Anna Jakubowska ${ }^{30,144}$, Paul James ${ }^{60}$, 145, Ramunas Janavicius ${ }^{146}$, Rachel C. Jankowitz ${ }^{147}$, Esther M. John ${ }^{148}$, Nichola Johnson ${ }^{97}$, Michael E. Jones $^{149}$, Arja Jukkola-Vuorinen ${ }^{150}$, Audrey Jung ${ }^{151}$, Rudolf Kaaks ${ }^{151}$, Daehee Kang ${ }^{152-154}$, Beth Y. $\operatorname{Karlan}^{155}$, Renske Keeman ${ }^{73}$, Michael J. Kerin ${ }^{156}$, Elza Khusnutdinova ${ }^{29,}{ }^{157}$, Johanna I. Kiiski ${ }^{158}$, Judy Kirk $^{159}$, Cari M. Kitahara ${ }^{160}$, Yon-Dschun Ko ${ }^{161}$, Irene Konstantopoulou ${ }^{162}$, Veli-Matti Kosma ${ }^{127-129}$, Stella Koutros $^{163}$, Katerina Kubelka-Sabit ${ }^{164}$, Ava Kwong ${ }^{165-167}$, Kyriacos Kyriacou ${ }^{7}$, Yael Laitman ${ }^{102}$, Diether Lambrechts $^{168,169}$, Eunjung Lee ${ }^{123}$, Goska Leslie ${ }^{6}$, Jenny Lester ${ }^{155}$, Fabienne Lesueur ${ }^{70,}$ 71, 170, Annika Lindblom $^{171,172}$, Wing-Yee Lo ${ }^{43}$, 44, Jirong Long ${ }^{26}$, Artitaya Lophatananon ${ }^{173,174}$, Jennifer T. Loud ${ }^{117}$, Jan Lubiński $^{30}$, Robert J. Maclnnis ${ }^{82,111}$, Tom Maishman ${ }^{88,89}$, Enes Makalic ${ }^{82}$, Arto Mannermaa ${ }^{127-129}$, Mehdi Manoochehri ${ }^{126}$, Siranoush Manoukian ${ }^{24}$, Sara Margolin ${ }^{125,}{ }^{175}$, Maria Elena Martinez ${ }^{106,}{ }^{176}$, Keitaro Matsuo ${ }^{177,}{ }^{178}$, Tabea Maurer ${ }^{179}$, Dimitrios Mavroudis ${ }^{110}$, Rebecca Mayes ${ }^{1}$, Lesley McGuffog ${ }^{6}$, Catriona McLean $^{180}$, Noura Mebirouk ${ }^{69-71}$, Alfons Meindl ${ }^{181}$, Pooja Middha ${ }^{151,182}$, Nicola Miller ${ }^{156}$, Austin Miller ${ }^{183}$, 
Marco Montagna ${ }^{9}$, Fernando Moreno ${ }^{56}$, Anna Marie Mulligan ${ }^{184,}{ }^{185}$, Victor M. Muñoz-Garzon ${ }^{186}$, Taru A. Muranen ${ }^{158}$, Steven A. Narod ${ }^{187}$, Rami Nassir ${ }^{188}$, Katherine L. Nathanson ${ }^{83}$, Susan L. Neuhausen ${ }^{81}$, Heli Nevanlinna ${ }^{158}$, Patrick Neven ${ }^{98}$, Finn C. Nielsen ${ }^{189}$, Liene Nikitina-Zake ${ }^{190}$, Aaron Norman ${ }^{191}$, Kenneth Offit $^{192,193}$, Edith Olah ${ }^{194}$, Olufunmilayo I. Olopade ${ }^{195}$, Håkan Olsson ${ }^{196}$, Nick Orr ${ }^{197}$, Ana Osorio ${ }^{27,} 28$, V. Shane Pankratz ${ }^{198}$, Janos Papp ${ }^{194}$, Sue K. Park ${ }^{152-154}$, Tjoung-Won Park-Simon ${ }^{36}$, Michael T. Parsons ${ }^{5}$, James Paul ${ }^{199}$, Inge Sokilde Pedersen ${ }^{200}$, Bernard Peissel ${ }^{24}$, Beth Peshkin ${ }^{141}$, Paolo Peterlongo ${ }^{201}$, Julian Peto $^{84}$, Dijana Plaseska-Karanfilska ${ }^{143}$, Karolina Prajzendanz ${ }^{30}$, Ross Prentice ${ }^{22}$, Nadege Presneau ${ }^{90}$, Darya Prokofyeva ${ }^{157}$, Miquel Angel Pujana202, Katri Pylkäs ${ }^{203,204}$, Paolo Radice ${ }^{205}$, Susan J. Ramus 206,207 , Johanna Rantala 208 , Rohini Rau-Murthy ${ }^{193}$, Gad Rennert ${ }^{209}$, Harvey A. Risch ${ }^{210}$, Mark Robson ${ }^{193}$, Atocha Romero $^{211}$, Caroline Maria Rossing ${ }^{189}$, Emmanouil Saloustros ${ }^{212}$, Estela Sánchez-Herrero ${ }^{211}$, Dale P. Sandler ${ }^{213}$, Marta Santamariña27, 214, 215, Christobel Saunders ${ }^{216}$, Elinor J. Sawyer ${ }^{217}$, Maren T. Scheuner ${ }^{31}$, Daniel F. Schmidt ${ }^{82,218}$, Rita K. Schmutzler ${ }^{121,122}$, Andreas Schneeweiss ${ }^{54,}$ 219, Minouk J. Schoemaker ${ }^{149}$, Ben Schöttker ${ }^{18,220}$, Peter Schürmann ${ }^{36}$, Christopher Scott $^{191}$, Rodney J. Scott ${ }^{221-223}$, Leigha Senter ${ }^{224}$, Caroline MD Seynaeve ${ }^{132}$, Mitul Shah ${ }^{1}$, Priyanka Sharma ${ }^{225}$, Chen-Yang Shen ${ }^{226,227,}$ Xiao-Ou Shu ${ }^{26}$, Christian F. Singer ${ }^{228}$, Thomas P. Slavin ${ }^{229}$, Snezhana Smichkoska ${ }^{230}$, Melissa C. Southey ${ }^{86,}$ 231, John J. Spinelli ${ }^{232,233}$, Amanda B. Spurdle ${ }^{5}$, Jennifer Stone ${ }^{82,234}$, Dominique Stoppa-Lyonnet ${ }^{69,235,236}$, Christian Sutter ${ }^{237}$, Anthony J. Swerdlow ${ }^{149,238}$, Rulla M. Tamimi ${ }^{3,4}$ 239, Yen Yen Tan ${ }^{240}$, William J. Tapper $^{241}$, Jack A. Taylor 213,242 , Manuel R. Teixeira243, 244, Maria Tengström ${ }^{127,245,246}$, Soo H. Teo ${ }^{247,248,}$ Mary Beth Terry ${ }^{249}$, Alex Teulé250, Mads Thomassen ${ }^{251}$, Darcy L. Thull252, Maria Grazia Tibiletti ${ }^{253}$, Marc Tischkowitz ${ }^{101,254}$, Amanda E. Toland ${ }^{255}$, Rob A.E.M. Tollenaar ${ }^{256}$, Ian Tomlinson ${ }^{257}$, 258, Diana Torres ${ }^{47}$, 126, Gabriela Torres-Mejía 259 , Melissa A. Troester ${ }^{260}$, Nadine Tung ${ }^{261}$, Maria Tzardi ${ }^{262}$, Hans-Ulrich Ulmer $^{263}$, Celine M. Vachon ${ }^{264}$, Christi J. van Asperen ${ }^{265}$, Lizet E. van der Kolk ${ }^{134}$, Elizabeth J. van 
Rensburg ${ }^{266}$, Ana Vega 27, 214, 215, Alessandra Viel ${ }^{267}$, Joseph Vijai ${ }^{192,193}$, Maatje J. Vogel ${ }^{268}$, Qin Wang6 Barbara Wappenschmidt ${ }^{121,122}$, Clarice R. Weinberg ${ }^{269}$, Jeffrey N. Weitzel ${ }^{229}$, Camilla Wendt ${ }^{175}$, Hans Wildiers $^{98}$, Robert Winqvist ${ }^{203,204}$, Alicja Wolk ${ }^{124,270}$, Anna H. Wu ${ }^{123}$, Drakoulis Yannoukakos ${ }^{162}$, Yan Zhang $^{18,45}$, Wei Zheng ${ }^{26}$, Paul D.P. Pharoah ${ }^{1,6}$, Jenny Chang-Claude ${ }^{151,179}$, Montserrat García-Closas ${ }^{163,}$ 271, Marjanka K. Schmidt ${ }^{73,272}$, Roger L. Milne ${ }^{82,86,111}$, Vessela N. Kristensen ${ }^{273,274}$, Juliet D. French ${ }^{5}$, Stacey L. Edwards ${ }^{5}$, Antonis C. Antoniou ${ }^{6}$, Georgia Chenevix-Trench ${ }^{5,276}$, Jacques Simard ${ }^{8,276}$, Douglas F. Easton $^{1,6,276}$, Peter Kraft ${ }^{3,4,276,{ }^{*}}$, Alison M. Dunning ${ }^{1,276,{ }^{*}}$

1 Centre for Cancer Genetic Epidemiology, Department of Oncology, University of Cambridge, Cambridge, CB1 8RN, UK.

${ }^{2}$ Centre de Bioinformatique, Biostatistique et Biologie Intégrative (C3BI), Institut Pasteur, Paris, France.

${ }^{3}$ Program in Genetic Epidemiology and Statistical Genetics, Harvard T.H. Chan School of Public Health, Boston, MA, 02115, USA.

${ }^{4}$ Department of Epidemiology, Harvard T.H. Chan School of Public Health, Boston, MA, 02115, USA.

${ }^{5}$ Department of Genetics and Computational Biology, QIMR Berghofer Medical Research Institute, Brisbane, Queensland, 4006, Australia.

${ }^{6}$ Centre for Cancer Genetic Epidemiology, Department of Public Health and Primary Care, University of Cambridge, Cambridge, CB1 8RN, UK.

7 Department of Electron Microscopy/Molecular Pathology and The Cyprus School of Molecular Medicine, The Cyprus Institute of Neurology \& Genetics, Nicosia, 1683, Cyprus.

${ }^{8}$ Genomics Center, Centre Hospitalier Universitaire de Québec - Université Laval, Research Center, Québec City, QC, G1V 4G2, Canada. 
${ }^{9}$ Immunology and Molecular Oncology Unit, Veneto Institute of Oncology IOV - IRCCS, Padua, 35128, Italy.

${ }^{10}$ Department of Clinical Genetics, Helsinki University Hospital, University of Helsinki, Helsinki, 00290, Finland.

${ }^{11}$ Human Genotyping-CEGEN Unit, Human Cancer Genetic Program, Spanish National Cancer Research Centre, Madrid, 28029, Spain.

12 Fred A. Litwin Center for Cancer Genetics, Lunenfeld-Tanenbaum Research Institute of Mount Sinai Hospital, Toronto, ON, M5G 1X5, Canada.

${ }^{13}$ Department of Molecular Genetics, University of Toronto, Toronto, ON, M5S 1A8, Canada.

${ }^{14}$ Department of Epidemiology, Genetic Epidemiology Research Institute, University of California Irvine, Irvine, CA, 92617, USA.

${ }^{15}$ N.N. Alexandrov Research Institute of Oncology and Medical Radiology, Minsk, 223040, Belarus.

${ }^{16}$ Department of Pathology, Landspitali University Hospital, Reykjavik, 101, Iceland.

${ }^{17}$ BMC (Biomedical Centre), Faculty of Medicine, University of Iceland, Reykjavik, 101, Iceland.

${ }^{18}$ Division of Clinical Epidemiology and Aging Research, C070, German Cancer Research Center (DKFZ), Heidelberg, 69120, Germany.

${ }^{19}$ Department of Public Health Sciences, and Cancer Research Institute, QueenÕs University, Kingston, ON, K7L 3N6, Canada.

${ }^{20}$ Department of Breast Medical Oncology, University of Texas MD Anderson Cancer Center, Houston, TX, 77030, USA.

21 Institute of Human Genetics, Hannover Medical School, Hannover, 30625, Germany.

${ }^{22}$ Cancer Prevention Program, Fred Hutchinson Cancer Research Center, Seattle, WA, 98109, USA. 
${ }^{23}$ Zilber School of Public Health, University of Wisconsin-Milwaukee, Milwaukee, WI, 53205, USA.

24 Unit of Medical Genetics, Department of Medical Oncology and Hematology, Fondazione IRCCS

(Istituto di Ricovero e Cura a Carattere Scientifico) Istituto Nazionale dei Tumori di Milano, Milan, 20133, Italy.

${ }^{25}$ High Risk and Cancer Prevention Group, Vall d'Hebron Institute of Oncology, Barcelona, 08035, Spain.

26 Division of Epidemiology, Department of Medicine, Vanderbilt Epidemiology Center, VanderbiltIngram Cancer Center, Vanderbilt University School of Medicine, Nashville, TN, 37232, USA.

${ }^{27}$ Centro de Investigación en Red de Enfermedades Raras (CIBERER), Valencia, 46010, Spain.

28 Human Cancer Genetics Programme, Spanish National Cancer Research Centre (CNIO), Madrid, 28029, Spain.

${ }^{29}$ Institute of Biochemistry and Genetics, Ufa Scientific Center of Russian Academy of Sciences, Ufa, 450054, Russia.

${ }^{30}$ Department of Genetics and Pathology, Pomeranian Medical University, Szczecin, 71-252, Poland.

${ }^{31}$ Cancer Genetics and Prevention Program, University of California San Francisco, San Francisco, CA, 94143-1714, USA.

${ }^{32}$ Department of Oncology, Helsinki University Hospital, University of Helsinki, Helsinki, 00290, Finland.

${ }^{33}$ Department of Oncology, Örebro University Hospital, Örebro, 70185, Sweden.

${ }^{34}$ International Epidemiology Institute, Rockville, MD, 20850, USA.

${ }^{35}$ Department of Radiation Oncology, Hannover Medical School, Hannover, 30625, Germany.

${ }^{36}$ Gynaecology Research Unit, Hannover Medical School, Hannover, 30625, Germany.

37 Copenhagen General Population Study, Herlev and Gentofte Hospital, Copenhagen University Hospital, Herlev, 2730, Denmark. 
${ }^{38}$ Department of Clinical Biochemistry, Herlev and Gentofte Hospital, Copenhagen University Hospital, Herlev, 2730, Denmark.

${ }^{39}$ Faculty of Health and Medical Sciences, University of Copenhagen, Copenhagen, 2200, Denmark.

${ }^{40}$ Division of Cancer Prevention and Genetics, IEO, European Institute of Oncology IRCCS, Milan, 20141, Italy.

${ }^{41}$ Department of Oncology, Lund University and Skåne University Hospital, Lund, 222 41, Sweden.

42 Institute of Medical Genetics and Applied Genomics, University of Tübingen, Tübingen, 72074, Germany.

${ }^{43}$ Dr. Margarete Fischer-Bosch-Institute of Clinical Pharmacology, Stuttgart, 70376, Germany.

44 University of Tübingen, Tübingen, 72074, Germany.

45 German Cancer Consortium (DKTK), German Cancer Research Center (DKFZ), Heidelberg, 69120, Germany.

46 Division of Preventive Oncology, German Cancer Research Center (DKFZ) and National Center for Tumor Diseases (NCT), Heidelberg, 69120, Germany.

${ }^{47}$ Institute of Human Genetics, Pontificia Universidad Javeriana, Bogota, Colombia.

${ }^{48}$ Medical Faculty, Universidad de La Sabana, Bogota, Colombia.

${ }^{49}$ Sheffield Institute for Nucleic Acids (SInFoNiA), Department of Oncology and Metabolism, University of Sheffield, Sheffield, S10 2TN, UK.

${ }^{50}$ Genome Sciences Centre, BC Cancer Agency, Vancouver, BC, V5Z 1L3, Canada.

${ }^{51}$ Department of Biomedical Physiology and Kinesiology, Simon Fraser University, Burnaby, BC, V5A 1S6, Canada. 
52 Institute for Prevention and Occupational Medicine of the German Social Accident Insurance, Institute of the Ruhr University Bochum (IPA), Bochum, 44789, Germany.

${ }^{53}$ Molecular Epidemiology Group, C080, German Cancer Research Center (DKFZ), Heidelberg, 69120, Germany.

${ }^{54}$ Molecular Biology of Breast Cancer, University Womens Clinic Heidelberg, University of Heidelberg, Heidelberg, 69120, Germany.

${ }^{55}$ Department of Medicine, Huntsman Cancer Institute, Salt Lake City, UT, 84112, USA.

56 Medical Oncology Department, Hospital Clínico San Carlos, Instituto de Investigación Sanitaria San Carlos (IdISSC), Centro Investigación Biomédica en Red de Cáncer (CIBERONC), Madrid, 28040, Spain.

57 Section of Molecular Genetics, Dept. of Laboratory Medicine, University Hospital of Pisa, Pisa, Italy.

${ }^{58}$ Department of Internal Medicine, Huntsman Cancer Institute, Salt Lake City, UT, 84112, USA.

${ }^{59}$ Research Department, Peter MacCallum Cancer Center, Melbourne, Victoria, 3000, Australia.

60 Sir Peter MacCallum Department of Oncology, The University of Melbourne, Melbourne, Victoria, 3000, Australia.

${ }^{61}$ Genomic Epidemiology Group, German Cancer Research Center (DKFZ), Heidelberg, 69120, Germany.

62 Cancer Research UK Cambridge Research Institute, Li Ka Shing Centre, University of Cambridge, Cambridge, UK.

63 Epidemiology Research Program, American Cancer Society, Atlanta, GA, 30303, USA.

${ }^{64}$ Oncology and Genetics Unit, Instituto de Investigacion Sanitaria Galicia Sur (IISGS), Xerencia de Xestion Integrada de Vigo-SERGAS, Vigo, 36312, Spain.

${ }^{65}$ CRCHU de Québec-oncologie, Québec, QC, G1S 4L8, Canada.

${ }^{66}$ Departments of Pediatrics and Medicine, Columbia University, New York, NY, 10032, USA. 
${ }^{67}$ Centre for Medical Genetics, Ghent University, Gent, 9000, Belgium.

68 Westmead Institute for Medical Research, University of Sydney, Sydney, New South Wales, 2145, Australia.

69 Department of Tumour Biology, INSERM U830, Paris, 75005, France.

${ }^{70}$ Institut Curie, Paris, 75005, France.

${ }^{71}$ Mines ParisTech, Fontainebleau, 77305, France.

72 Department of Clinical Genetics, Erasmus University Medical Center, Rotterdam, 3015 CN, The Netherlands.

73 Division of Molecular Pathology, The Netherlands Cancer Institute - Antoni van Leeuwenhoek Hospital, Amsterdam, 1066 CX, The Netherlands.

74 Department of Laboratory Medicine and Pathology, Mayo Clinic, Rochester, MN, 55905, USA.

${ }^{75}$ Academic Unit of Pathology, Department of Neuroscience, University of Sheffield, Sheffield, S10 2TN, UK.

76 Department of Medical Epidemiology and Biostatistics, Karolinska Institutet, Stockholm, 171 65, Sweden.

77 Department of Clinical Genetics, Fox Chase Cancer Center, Philadelphia, PA, 19111, USA.

78 Department of Pathology, Leiden University Medical Center, Leiden, 2333 ZA, The Netherlands.

${ }^{79}$ Department of Human Genetics, Leiden University Medical Center, Leiden, 2333 ZA, The Netherlands.

80 Department of Medical Oncology, Vall d'Hebron Institute of Oncology (VHIO), University Hospital, Vall d'Hebron, Barcelona, 08035, Spain.

${ }^{81}$ Department of Population Sciences, Beckman Research Institute of City of Hope, Duarte, CA, 91010, USA. 
${ }^{82}$ Centre for Epidemiology and Biostatistics, Melbourne School of Population and Global Health, The University of Melbourne, Melbourne, Victoria, 3010, Australia.

${ }^{83}$ Department of Medicine, Abramson Cancer Center, Perelman School of Medicine at the University of Pennsylvania, Philadelphia, PA, 19104, USA.

${ }^{84}$ Department of Non-Communicable Disease Epidemiology, London School of Hygiene and Tropical Medicine, London, WC1E 7HT, UK.

85 Département de Médecine Moléculaire, Faculté de Médecine, Centre Hospitalier Universitaire de Québec Research Center, Laval University, Québec City, QC, G1V 0A6, Canada.

86 Precision Medicine, School of Clinical Sciences at Monash Health, Monash University, Clayton, Victoria, 3168, Australia.

87 Cáncer Hereditario, Instituto de Biología y Genética Molecular, IBGM, Universidad de Valladolid, Centro Superior de Investigaciones Cient'ficas, UVA-CSIC, Valladolid, 47003, Spain.

88 Southampton Clinical Trials Unit, Faculty of Medicine, University of Southampton, Southampton, SO17 1BJ, UK.

${ }^{89}$ Cancer Sciences Academic Unit, Faculty of Medicine, University of Southampton, Southampton, SO17 1BJ, UK.

${ }^{90}$ Department of Biomedical Sciences, Faculty of Science and Technology, University of Westminster, London, W1B 2HW, UK.

91 Institute for Medical Informatics, Statistics and Epidemiology, University of Leipzig, Leipzig, 04107, Germany.

92 LIFE - Leipzig Research Centre for Civilization Diseases, University of Leipzig, Leipzig, 04103, Germany. 
93 Division of Evolution and Genomic Medicine, School of Biological Sciences, Faculty of Biology, Medicine and Health, University of Manchester, Manchester Academic Health Science Centre, Manchester, M13 9WL, UK.

${ }^{94}$ Manchester Centre for Genomic Medicine, St Mary's Hospital, Manchester University Hospitals NHS Foundation Trust, Manchester Academic Health Science Centre, Manchester, M13 9WL, UK.

95 David Geffen School of Medicine, Department of Medicine Division of Hematology and Oncology, University of California at Los Angeles, Los Angeles, CA, 90095, USA.

96 Department of Gynecology and Obstetrics, Comprehensive Cancer Center ER-EMN, University Hospital Erlangen, Friedrich-Alexander-University Erlangen-Nuremberg, Erlangen, 91054, Germany.

97 The Breast Cancer Now Toby Robins Research Centre, The Institute of Cancer Research, London, SW7 3RP, UK.

${ }^{98}$ Leuven Multidisciplinary Breast Center, Department of Oncology, Leuven Cancer Institute, University Hospitals Leuven, Leuven, 3000, Belgium.

99 Department of Breast Surgery, Herlev and Gentofte Hospital, Copenhagen University Hospital, Herlev, 2730, Denmark.

100 Department of Cancer Epidemiology and Genetics, Masaryk Memorial Cancer Institute, Brno, 65653, Czech Republic.

101 Program in Cancer Genetics, Departments of Human Genetics and Oncology, McGill University, MontrŽal, QC, H4A 3J1, Canada.

102 The Susanne Levy Gertner Oncogenetics Unit, Chaim Sheba Medical Center, Ramat Gan, 52621, Israel.

${ }^{103}$ Sackler Faculty of Medicine, Tel Aviv University, Ramat Aviv, 69978, Israel. 
${ }^{104}$ School of Public Health, Curtin University, Perth, Western Australia, 6102, Australia.

105 Genomic Medicine Group, Galician Foundation of Genomic Medicine, Instituto de Investigación Sanitaria de Santiago de Compostela (IDIS), Complejo Hospitalario Universitario de Santiago, SERGAS, Santiago de Compostela, 15706, Spain.

106 Moores Cancer Center, University of California San Diego, La Jolla, CA, 92037, USA.

107 Section of Molecular Genetics, Department of Laboratory Medicine, University Hospital of Pisa, Pisa, Italy.

108 Schools of Medicine and Public Health, Division of Cancer Prevention \& Control Research, Jonsson Comprehensive Cancer Centre, UCLA, Los Angeles, CA, 90096-6900, USA.

${ }^{109}$ Cancer Risk and Prevention Clinic, Dana-Farber Cancer Institute, Boston, MA, 02215, USA.

${ }^{110}$ Department of Medical Oncology, University Hospital of Heraklion, Heraklion, 711 10, Greece.

${ }^{111}$ Cancer Epidemiology \& Intelligence Division, Cancer Council Victoria, Melbourne, Victoria, 3004, Australia.

112 Department of Epidemiology and Preventive Medicine, Monash University, Melbourne, Victoria, 3004, Australia.

113 Department of Pathology and Laboratory Medicine, Kansas University Medical Center, Kansas City, KS, 66160, USA.

${ }^{114}$ Department of Medicine, McGill University, Montréal, QC, H4A 3J1, Canada.

115 Division of Clinical Epidemiology, Royal Victoria Hospital, McGill University, Montréal, QC, H4A 3J1, Canada.

${ }^{116}$ Department of Dermatology, Huntsman Cancer Institute, University of Utah School of Medicine, Salt Lake City, UT, 84112, USA. 
117 Clinical Genetics Branch, Division of Cancer Epidemiology and Genetics, National Cancer Institute, Bethesda, MD, 20850-9772, USA.

118 Department of Surgery, Oulu University Hospital, University of Oulu, Oulu, 90220, Finland.

119 Centre de Recherche du Centre Hospitalier de Université de Montréal (CHUM), Université de Montréal, Montréal, QC, H2X 0A9, Canada.

${ }^{120}$ Cancer \& Environment Group, Center for Research in Epidemiology and Population Health (CESP), INSERM, University Paris-Sud, University Paris-Saclay, Villejuif, 94805, France.

${ }^{121}$ Center for Hereditary Breast and Ovarian Cancer, University Hospital of Cologne, Cologne, 50937, Germany.

${ }^{122}$ Center for Molecular Medicine Cologne (CMMC), University of Cologne, Cologne, 50931, Germany.

${ }^{123}$ Department of Preventive Medicine, Keck School of Medicine, University of Southern California, LoS Angeles, CA, 90033, USA.

124 Institute of Environmental Medicine, Karolinska Institutet, Stockholm, 171 77, Sweden.

125 Department of Oncology, Södersjukhuset, Stockholm, 118 83, Sweden.

${ }^{126}$ Molecular Genetics of Breast Cancer, German Cancer Research Center (DKFZ), Heidelberg, 69120, Germany.

${ }^{127}$ Translational Cancer Research Area, University of Eastern Finland, Kuopio, 70210, Finland.

128 Institute of Clinical Medicine, Pathology and Forensic Medicine, University of Eastern Finland, Kuopio, 70210, Finland.

129 Imaging Center, Department of Clinical Pathology, Kuopio University Hospital, Kuopio, 70210, Finland. 
130 Saw Swee Hock School of Public Health, National University of Singapore, Singapore, 119077, Singapore.

${ }^{131}$ Department of Surgery, National University Health System, Singapore, 119228, Singapore.

132 Department of Medical Oncology, Family Cancer Clinic, Erasmus MC Cancer Institute, Rotterdam, 3015 CN, The Netherlands.

133 School of Population and Global Health, The University of Western Australia, Perth, Western Australia, 6009, Australia.

134 Family Cancer Clinic, The Netherlands Cancer Institute - Antoni van Leeuwenhoek hospital, Amsterdam, 1066 CX, The Netherlands.

135 Division of Cancer Sciences, University of Manchester, Manchester, M13 9PL, UK.

${ }^{136}$ Center for Medical Genetics, NorthShore University HealthSystem, Evanston, IL, 60201, USA.

137 The University of Chicago Pritzker School of Medicine, Chicago, IL, 60637, USA.

138 N.N. Petrov Institute of Oncology, St. Petersburg, 197758, Russia.

139 Australian Breast Cancer Tissue Bank, Westmead Institute for Medical Research, University of Sydney, Sydney, New South Wales, 2145, Australia.

140 The Hereditary Breast and Ovarian Cancer Research Group Netherlands (HEBON), Coordinating center: The Netherlands Cancer Institute, Amsterdam, 1066 CX, The Netherlands.

${ }^{141}$ Lombardi Comprehensive Cancer Center, Georgetown University, Washington, DC, 20007, USA. 142 Division of Epidemiology, Center for Public Health Sciences, National Cancer Center, Tokyo, 1040045, Japan.

143 Research Centre for Genetic Engineering and Biotechnology 'Georgi D. Efremov', Macedonian Academy of Sciences and Arts, Skopje, 1000, Republic of Macedonia. 
144 Independent Laboratory of Molecular Biology and Genetic Diagnostics, Pomeranian Medical University, Szczecin, 71-252, Poland.

145 Parkville Familial Cancer Centre, Peter MacCallum Cancer Center, Melbourne, Victoria, 3000, Australia.

146 Hematology, oncology and transfusion medicine center, Dept. of Molecular and Regenerative Medicine, Vilnius University Hospital Santariskiu Clinics, Vilnius, Lithuania.

147 Department of Medicine, Division of Hematology/Oncology, UPMC Hillman Cancer Center; University of Pittsburgh School of Medicine, Pittsburgh, PA 15232, USA.

${ }^{148}$ Department of Medicine, Division of Oncology, Stanford Cancer Institute, Stanford University School of Medicine, Stanford, CA, 94304, USA.

${ }^{149}$ Division of Genetics and Epidemiology, The Institute of Cancer Research, London, SM2 5NG, UK.

${ }^{150}$ Department of Oncology, Tampere University Hospital, Tampere, 33521, Finland.

151 Division of Cancer Epidemiology, German Cancer Research Center (DKFZ), Heidelberg, 69120, Germany.

152 Department of Preventive Medicine, Seoul National University College of Medicine, Seoul, 03080, Korea.

${ }^{153}$ Department of Biomedical Sciences, Seoul National University Graduate School, Seoul, 03080, Korea. ${ }^{154}$ Cancer Research Institute, Seoul National University, Seoul, 03080, Korea.

155 Women's Cancer Program at the Samuel Oschin Comprehensive Cancer Institute, Cedars-Sinai Medical Center, Los Angeles, CA, 90048, USA.

156 Surgery, School of Medicine, National University of Ireland, Galway, H91TK33, Ireland.

157 Department of Genetics and Fundamental Medicine, Bashkir State University, Ufa, 450076, Russia. 
158 Department of Obstetrics and Gynecology, Helsinki University Hospital, University of Helsinki, Helsinki, 00290, Finland.

${ }^{159}$ Familial Cancer Service, Weatmead Hospital, Wentworthville, New South Wales, 2145, Australia.

160 Radiation Epidemiology Branch, Division of Cancer Epidemiology and Genetics, National Cancer Institute, Bethesda, MD, 20892, USA.

161 Department of Internal Medicine, Evangelische Kliniken Bonn gGmbH, Johanniter Krankenhaus, Bonn, 53177, Germany.

162 Molecular Diagnostics Laboratory, INRASTES, National Centre for Scientific Research 'Demokritos', Athens, 15310, Greece.

163 Division of Cancer Epidemiology and Genetics, National Cancer Institute, National Institutes of Health, Department of Health and Human Services, Bethesda, MD, 20850, USA.

164 Department of Histopathology and Cytology, Clinical Hospital Acibadem Sistina, Skopje, 1000, Republic of Macedonia.

165 Hong Kong Hereditary Breast Cancer Family Registry, Cancer Genetics Centre, Happy Valley, Hong Kong.

166 Department of Surgery, The University of Hong Kong, Pok Fu Lam, Hong Kong.

167 Department of Surgery, Hong Kong Sanatorium and Hospital, Happy Valley, Hong Kong.

${ }^{168}$ VIB Center for Cancer Biology, VIB, Leuven, 3001, Belgium.

${ }^{169}$ Laboratory for Translational Genetics, Department of Human Genetics, University of Leuven, Leuven, 3000, Belgium.

170 Genetic Epidemiology of Cancer team, Inserm U900, Paris, 75005, France.

${ }^{171}$ Department of Molecular Medicine and Surgery, Karolinska Institutet, Stockholm, 171 76, Sweden. 
172 Department of Clinical Genetics, Karolinska University Hospital, Stockholm, 171 76, Sweden.

173 Division of Health Sciences, Warwick Medical School, University of Warwick, Coventry, CV4 7AL, UK.

174 Institute of Population Health, University of Manchester, Manchester, M13 9PL, UK.

175 Department of Clinical Science and Education, Sšdersjukhuset, Karolinska Institutet, Stockholm, 118

83, Sweden.

176 Department of Family Medicine and Public Health, University of California San Diego, La Jolla, CA, 92093, USA.

177 Division of Molecular Medicine, Aichi Cancer Center Research Institute, Nagoya, 464-8681, Japan.

178 Department of Epidemiology, Nagoya University Graduate School of Medicine, Nagoya, 466-8550, Japan.

${ }^{179}$ Cancer Epidemiology Group, University Cancer Center Hamburg (UCCH), University Medical Center Hamburg-Eppendorf, Hamburg, 20246, Germany.

${ }^{180}$ Anatomical Pathology, The Alfred Hospital, Melbourne, Victoria, 3004, Australia.

${ }^{181}$ Department of Gynecology and Obstetrics, Ludwig Maximilian University of Munich, Munich, 80336, Germany.

182 Faculty of Medicine, University of Heidelberg, Heidelberg, 69120, Germany.

183 NRG Oncology, Statistics and Data Management Center, Roswell Park Cancer Institute, Buffalo, NY, 14263, USA.

${ }^{184}$ Department of Laboratory Medicine and Pathobiology, University of Toronto, Toronto, ON, M5S 1A8, Canada.

185 Laboratory Medicine Program, University Health Network, Toronto, ON, M5G 2C4, Canada.

${ }^{186}$ Radiation Oncology, Hospital Meixoeiro-XXI de Vigo, Vigo, 36214, Spain. 
${ }^{187}$ Women's College Research Institute, University of Toronto, Toronto, ON, M5S 1A8, Canada.

188 Department of Biochemistry and Molecular Medicine, University of California Davis, Davis, CA, 95817, USA.

189 Center for Genomic Medicine, Rigshospitalet, Copenhagen University Hospital, Copenhagen, DK2100, Denmark.

190 Latvian Biomedical Research and Study Centre, Riga, Latvia.

191 Department of Health Sciences Research, Mayo Clinic, Rochester, MN, 55905, USA.

192 Clinical Genetics Research Lab, Department of Cancer Biology and Genetics, Memorial SloanKettering Cancer Center, New York, NY, 10065, USA.

193 Clinical Genetics Service, Department of Medicine, Memorial Sloan-Kettering Cancer Center, New York, NY, 10065, USA.

${ }^{194}$ Department of Molecular Genetics, National Institute of Oncology, Budapest, 1122, Hungary.

${ }^{195}$ Center for Clinical Cancer Genetics, The University of Chicago, Chicago, IL, 60637, USA.

196 Department of Cancer Epidemiology, Clinical Sciences, Lund University, Lund, 222 42, Sweden.

197 Centre for Cancer Research and Cell Biology, Queen's University Belfast, Belfast, Ireland, BT7 1NN, UK.

198 University of New Mexico Health Sciences Center, University of New Mexico, Albuquerque, NM, 87131, USA.

${ }^{199}$ Cancer Research UK Clinical Trials Unit, Institute of Cancer Sciences, University of Glasgow, Glasgow, G12 OYN, UK.

200 Section of Molecular Diagnostics, Clinical Biochemistry, Aalborg University Hospital, Aalborg, 9000, Denmark. 
${ }^{201}$ Genome Diagnostics Program, IFOM - the FIRC (Italian Foundation for Cancer Research) Institute of Molecular Oncology, Milan, 20139, Italy.

202 Translational Research Laboratory, IDIBELL (Bellvitge Biomedical Research Institute), Catalan Institute of Oncology, CIBERONC, Barcelona, 08908, Spain.

${ }^{203}$ Laboratory of Cancer Genetics and Tumor Biology, Cancer and Translational Medicine Research Unit, Biocenter Oulu, University of Oulu, Oulu, 90570, Finland.

204 Laboratory of Cancer Genetics and Tumor Biology, Northern Finland Laboratory Centre Oulu, Oulu, 90570, Finland.

205 Unit of Molecular Bases of Genetic Risk and Genetic Testing, Department of Research, Fondazione IRCCS (Istituto Di Ricovero e Cura a Carattere Scientifico) Istituto Nazionale dei Tumori (INT), Milan, 20133, Italy.

${ }^{206}$ School of Women's and Children's Health, Faculty of Medicine, University of NSW Sydney, Sydney, New South Wales, 2052, Australia.

207 The Kinghorn Cancer Centre, Garvan Institute of Medical Research, Sydney, New South Wales, 2010, Australia.

${ }^{208}$ Clinical Genetics, Karolinska Institutet, Stockholm, 171 76, Sweden.

${ }^{209}$ Clalit National Cancer Control Center, Carmel Medical Center and Technion Faculty of Medicine, Haifa, 35254, Israel.

${ }^{210}$ Chronic Disease Epidemiology, Yale School of Public Health, New Haven, CT, 06510, USA.

${ }^{211}$ Medical Oncology Department, Hospital Universitario Puerta de Hierro, Madrid, 28222, Spain.

212 Department of Oncology, University Hospital of Larisa, Larisa, 411 10, Greece. 
213 Epidemiology Branch, National Institute of Environmental Health Sciences, NIH, Research Triangle Park, NC, 27709, USA.

${ }^{214}$ Fundación Pública Galega Medicina Xenómica, Santiago De Compostela, 15706, Spain.

215 Instituto de Investigación Sanitaria de Santiago de Compostela, Santiago De Compostela, 15706, Spain.

${ }^{216}$ School of Medicine, University of Western Australia, Perth, Western Australia, Australia.

${ }^{217}$ Research Oncology, Guy's Hospital, King's College London, London, SE1 9RT, UK.

${ }^{218}$ Faculty of Information Technology, Monash University, Melbourne, Victoria, 3800, Australia.

219 National Center for Tumor Diseases, University Hospital and German Cancer Research Center, Heidelberg, 69120, Germany.

220 Network Aging Research, University of Heidelberg, Heidelberg, 6915, Germany.

${ }^{221}$ Division of Molecular Medicine, Pathology North, John Hunter Hospital, Newcastle, New South Wales, 2305, Australia.

222 Discipline of Medical Genetics, School of Biomedical Sciences and Pharmacy, Faculty of Health, University of Newcastle, Callaghan, New South Wales, 2308, Australia.

223 Hunter Medical Research Institute, John Hunter Hospital, Newcastle, New South Wales, 2305, Australia.

${ }^{224}$ Clinical Cancer Genetics Program, Division of Human Genetics, Department of Internal Medicine, The Comprehensive Cancer Center, The Ohio State University, Columbus, OH, 43210, USA.

225 Department of Internal Medicine, Division of Oncology, University of Kansas Medical Center, Westwood, KS, 66205, USA.

${ }^{226}$ Institute of Biomedical Sciences, Academia Sinica, Taipei, 115, Taiwan. 
227 School of Public Health, China Medical University, Taichung, Taiwan.

${ }^{228}$ Dept of OB/GYN and Comprehensive Cancer Center, Medical University of Vienna, Vienna, 1090, Austria.

${ }^{229}$ Clinical Cancer Genetics, City of Hope, Duarte, CA, 91010, USA.

${ }^{230}$ Ss. Cyril and Methodius University in Skopje, Medical Faculty, University Clinic of Radiotherapy and Oncology, Skopje, 1000, Republic of Macedonia.

231 Department of Clinical Pathology, The University of Melbourne, Melbourne, Victoria, 3010, Australia.

232 Population Oncology, BC Cancer, Vancouver, BC, V5Z 1G1, Canada.

233 School of Population and Public Health, University of British Columbia, Vancouver, BC, V6T 1Z4, Canada.

${ }^{234}$ The Curtin UWA Centre for Genetic Origins of Health and Disease, Curtin University and University of Western Australia, Perth, Western Australia, 6000, Australia.

235 Service de Génétique, Institut Curie, Paris, 75005, France.

236 Université Paris Descartes, Paris, 75006, France.

${ }^{237}$ Institute of Human Genetics, University Hospital Heidelberg, Heidelberg, 69120, Germany.

${ }^{238}$ Division of Breast Cancer Research, The Institute of Cancer Research, London, SW7 3RP, UK.

${ }^{239}$ Channing Division of Network Medicine, Department of Medicine, Brigham and Women's Hospital, Harvard Medical School, Boston, MA, 02115, USA.

${ }^{240}$ Dept of OB/GYN, Medical University of Vienna, Vienna, 1090, Austria.

${ }^{241}$ Faculty of Medicine, University of Southampton, Southampton, SO17 1BJ, UK. 
242 Epigenetic and Stem Cell Biology Laboratory, National Institute of Environmental Health Sciences, NIH, Research Triangle Park, NC, 27709, USA.

${ }^{243}$ Department of Genetics, Portuguese Oncology Institute, Porto, 4220-072, Portugal.

244 Biomedical Sciences Institute (ICBAS), University of Porto, Porto, 4050-013, Portugal.

${ }^{245}$ Cancer Center, Kuopio University Hospital, Kuopio, 70210, Finland.

246 Institute of Clinical Medicine, Oncology, University of Eastern Finland, Kuopio, 70210, Finland.

247 Cancer Research Malaysia, Subang Jaya, Selangor, 47500, Malaysia.

248 Breast Cancer Research Unit, Cancer Research Institute, University Malaya Medical Centre, Kuala Lumpur, 59100, Malaysia.

249 Department of Epidemiology, Mailman School of Public Health, Columbia University, New York, NY, 10032, USA.

250 Genetic Counseling Unit, Hereditary Cancer Program, IDIBELL (Bellvitge Biomedical Research Institute), Catalan Institute of Oncology, CIBERONC, Barcelona, 08908, Spain.

${ }^{251}$ Department of Clinical Genetics, Odense University Hospital, Odence C, 5000, Denmark.

252 Department of Medicine, Magee-Womens Hospital, University of Pittsburgh School of Medicine, Pittsburgh, PA, 15213, USA.

253 UO Anatomia Patologica, Ospedale di Circolo-Universit^ dell'Insubria, Varese, 21100, Italy.

254 Department of Medical Genetics, University of Cambridge, Cambridge, CB2 OQQ, UK.

255 Department of Cancer Biology and Genetics, The Ohio State University, Columbus, OH, 43210, USA.

${ }^{256}$ Department of Surgery, Leiden University Medical Center, Leiden, 2333 ZA, The Netherlands.

257 Institute of Cancer and Genomic Sciences, University of Birmingham, Birmingham, B15 2TT, UK. 
${ }^{258}$ Wellcome Trust Centre for Human Genetics and Oxford NIHR Biomedical Research Centre, University of Oxford, Oxford, OX3 7BN, UK.

${ }^{259}$ Center for Population Health Research, National Institute of Public Health, Mexico, 62100, Mexico. 260 Department of Epidemiology, Gilliungs School of Global Public Health and UNC Lineberger Comprehensive Cancer Center, University of North Carolina at Chapel Hill, Chapel Hill, NC, USA.

${ }^{261}$ Department of Medical Oncology, Beth Israel Deaconess Medical Center, Boston, MA, 02215, USA.

262 Department of Pathology, University Hospital of Heraklion, Heraklion, 711 10, Greece.

${ }^{263}$ Frauenklinik der Stadtklinik Baden-Baden, Baden-Baden, 76532, Germany.

264 Department of Health Science Research, Division of Epidemiology, Mayo Clinic, Rochester, MN, 55905, USA.

265 Department of Clinical Genetics, Leiden University Medical Center, Leiden, 2333 ZA, The Netherlands.

266 Department of Genetics, University of Pretoria, Arcadia, 0007, South Africa.

267 Division of Functional onco-genomics and genetics, Centro di Riferimento Oncologico di Aviano (CRO), IRCCS, Aviano, 33081, Italy.

268 Department of Pathology, Netherlands Cancer Institute, 1006 BE,

${ }^{269}$ Biostatistics and Computational Biology Branch, National Institute of Environmental Health Sciences, NIH, Research Triangle Park, NC, 27709, USA.

270 Department of Surgical Sciences, Uppsala University, Uppsala, 751 05, Sweden.

${ }^{271}$ Division of Genetics and Epidemiology, Institute of Cancer Research, London, SM2 5NG, UK.

272 Division of Psychosocial Research and Epidemiology, The Netherlands Cancer Institute - Antoni van Leeuwenhoek hospital, Amsterdam, 1066 CX, The Netherlands. 
273 Department of Cancer Genetics, Institute for Cancer Research, Oslo University HospitalRadiumhospitalet, Oslo, 0379, Norway.

274 Institute of Clinical Medicine, Faculty of Medicine, University of Oslo, Oslo, 0450, Norway.

275 These authors contributed equally.

${ }^{276}$ Senior author.

* Correspondence: pkraft@hsph.harvard.edu (PK), amd24@medschl.cam.ac.uk (AMD)

\section{ABSTRACT}

Genome-wide association studies have identified breast cancer risk variants in over 150 genomic regions, but the mechanisms underlying risk remain largely unknown. These regions were explored by combining association analysis with in silico genomic feature annotations. We defined 205 independent risk-associated signals with the set of credible causal variants (CCVs) in each one. In parallel, we used a Bayesian approach (PAINTOR) that combines genetic association, linkage disequilibrium, and enriched genomic features to determine variants with high posterior probabilities (HPPs) of being causal. Potentially causal variants were significantly over-represented in active gene regulatory regions and transcription factor binding sites. We applied our INQUSIT pipeline for prioritizing genes as targets of potentially causal variants, using gene expression (eQTL), chromatin interaction and functional annotations. Known cancer drivers, transcription factors and genes in the developmental, apoptosis, immune system and DNA integrity checkpoint gene ontology pathways, were over-represented among the 178 highest confidence target genes. 


\section{INTRODUCTION}

Genome-wide association studies (GWAS) have identified genetic variants associated with breast cancer risk in more than 150 genomic regions ${ }^{1,2}$. However, the variants and genes driving these associations are mostly unknown, with fewer than 20 regions studied in detail ${ }^{3-20}$. Here, we aimed to fine-map all known breast cancer susceptibility regions using dense genotype data on > 217K subjects participating in the Breast Cancer Association Consortium (BCAC) and the Consortium of Investigators of Modifiers of BRCA1/2 (CIMBA). All samples were genotyped using the OncoArray ${ }^{\top \mathrm{M} 1,2,21}$ or the iCOGS chip $^{22,23}$. Stepwise multinomial logistic regression was used to identify independent association signals in each region and define credible causal variants (CCVs) within each signal. We found genomic features significantly overlapping the CCVs and then used a Bayesian approach, integrating informative genomic features and genetic associations, to refine the set of likely causal variants and calculate their posterior probabilities. Finally, we integrated genetic and in silico epigenetic, expression and chromatin conformation data to infer the likely target genes of each signal.

\section{RESULTS}

\section{Most breast cancer genomic regions contain multiple independent risk-associated signals}

We included 109,900 breast cancer cases and 88,937 controls, all of European ancestry, from 75 studies in the BCAC. Genotypes (directly observed or imputed) were available for 639,118 single nucleotide polymorphisms (SNPs), deletion/insertions, and copy number variants (CNVs) with minor allele frequency $(\mathrm{MAF}) \geq 0.1 \%$ within 152, previously defined, risk-associated regions (Supplementary Table 1; Figure 1). Multivariate logistic regression confirmed associations for 150/152 regions at a $p$-value $<$ $10^{-4}$ significance threshold (Supplementary Table $2 \mathrm{~A}$ ). To determine the number of independent risk 


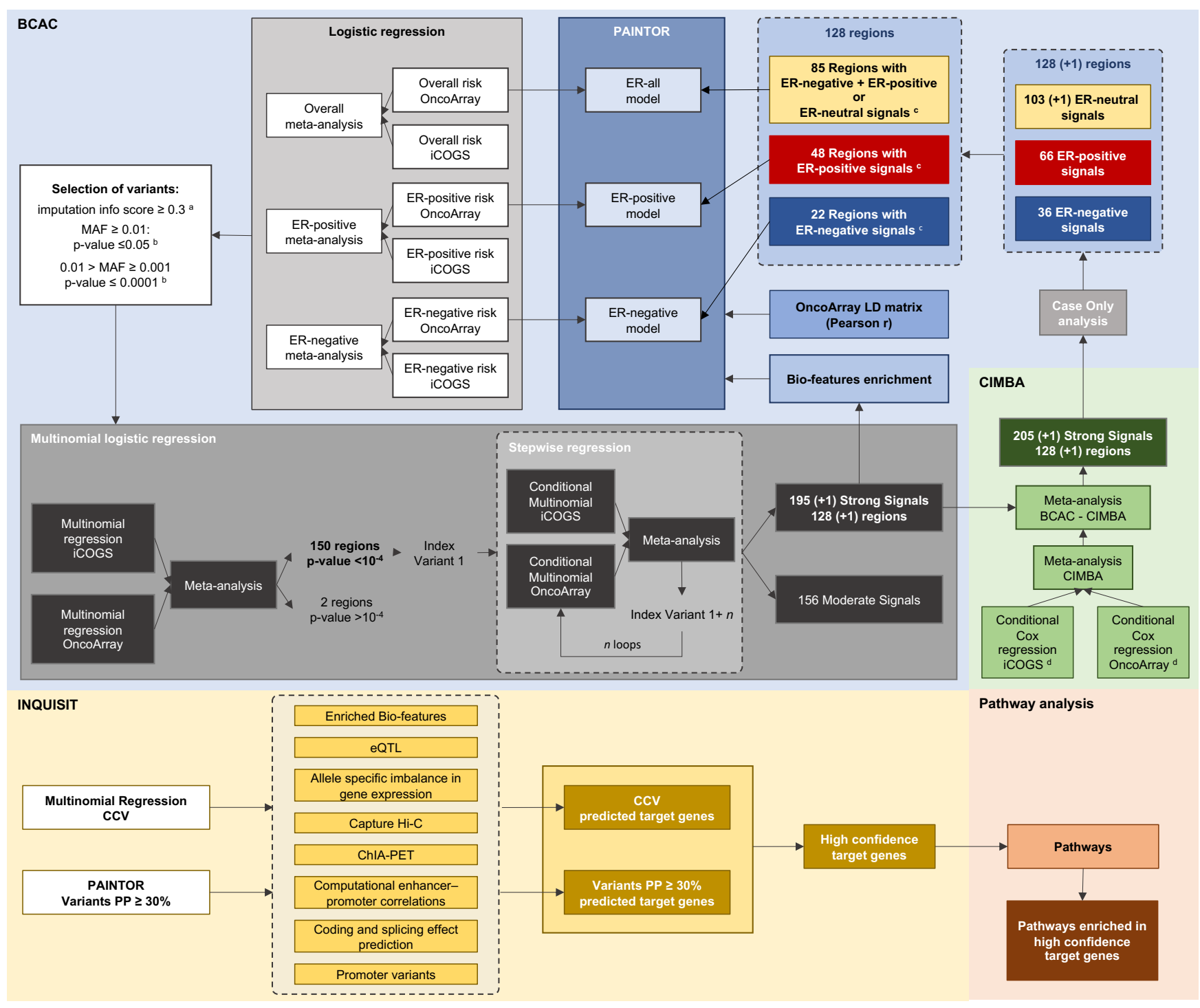

Figure 1. Flowchart summarizing the study design. ${ }^{a}$ In iCOGS and in OncoArray; ${ }^{b}$ for at least one phenotype; ${ }^{c}$ regions containing signals associated with more than one phenotype were evaluated with more than one PAINTOR model; ${ }^{d}$ conditional on the index variants from BCAC strong signals.

signals within each region we applied stepwise multinomial logistic regression, deriving the association of each variant, conditional on the more significant ones, in order of statistical significance. Finally, we defined CCVs in each signal as variants with conditional p-values within two orders of magnitude of the 
index variant ${ }^{24}$. We classified the evidence for each independent signal, and it's CCVs, as either strong (conditional $p$-values $\left.<10^{-6}\right)$ or moderate $\left(10^{-6}<\right.$ conditional $p$-values $\left.<10^{-4}\right)$.

From the 150 genomic regions we identified 352 independent risk signals containing 13,367 CCVs, 7,394 of these were within the 196 strong-evidence signals across 129 regions (Figures $\mathbf{2 A - B}$ ). The number of signals per region ranged from 1 to 9 , with 79 (53\%) containing multiple signals. We noted a wide range of CCVs per signal, but in 42 signals there was only a single CCV and this was assumed to be causal (Figures 2C-D, Table 1).

The majority of breast tumors express the estrogen receptor (ER-positive), but 20\% do not (ERnegative); these two tumor types have distinct biological and clinical characteristics ${ }^{25}$. Using a caseonly analysis for the 196 strong-evidence signals, we found 66 signals (34\%; containing 1,238 CCVs) where the lead variant conferred a greater relative-risk of developing ER-positive tumors (false discovery rate, FDR 5\%), and 29 (15\%; 646 CCVs) where the lead variant conferred a greater risk of ERnegative cancer tumors (FDR 5\%) (Supplementary Table 2B, Figure 2E). The remaining 101 signals (51\%, 5,510 CCVs) showed no difference by ER status (referred to as ER-neutral).

Patients with BRCA1 mutations are more likely to develop ER-negative tumors ${ }^{26}$. Hence, to increase our power to identify ER-negative signals, we performed a fixed-effects meta-analysis, combining association results from BRCA1 mutation carriers in CIMBA with the BCAC ER-negative association results. This meta-analysis identified ten additional signals, seven ER-negative and three ER-neutral, 
a

Number of

Regions

b

Number of

CCVs

c

Number of

Signals

d

Number of

Signals

e

Number of

CCVs

f

Number of

CCVs

g

Number of

Variants
Number of signals

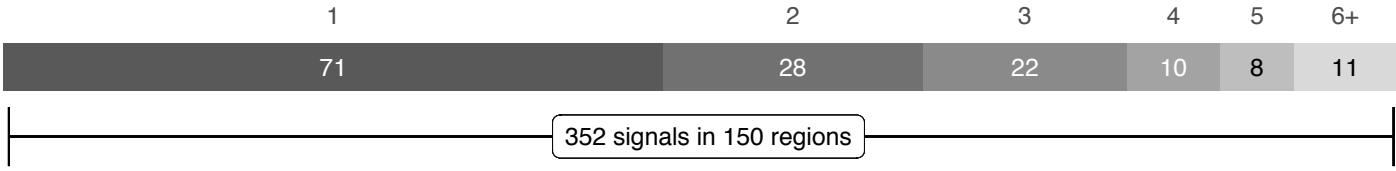

Signal classification

Strong confidence signals

Moderate confidence signals

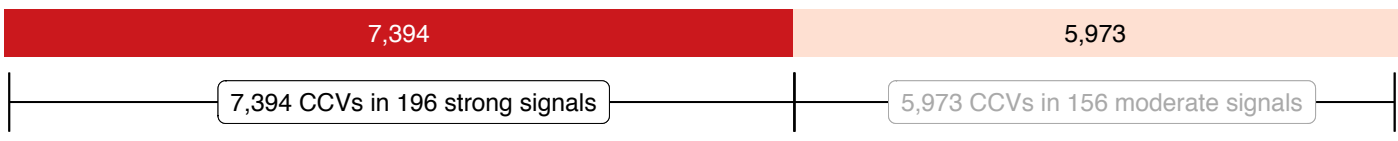

Number of CCVs

\begin{tabular}{|ccccccc|}
1 & $2-5$ & $6-10$ & $11-20$ & $21-30$ & $31-50$ & $51+$ \\
\hline 28 & 38 & 30 & 31 & 21 & 19 & 29 \\
\hline & & & & \\
\hline
\end{tabular}

Number of CCVs

\begin{tabular}{|c|c|c|c|c|c|c|}
\hline 1 & $2-5$ & $6-10$ & $11-20$ & $21-30$ & $31-50$ & $51+$ \\
\hline 14 & 23 & 19 & 17 & 22 & 21 & 40 \\
\hline
\end{tabular}

Signal phenotype classification

66 ER-positive 29 ER-negative

101 ER-neutral

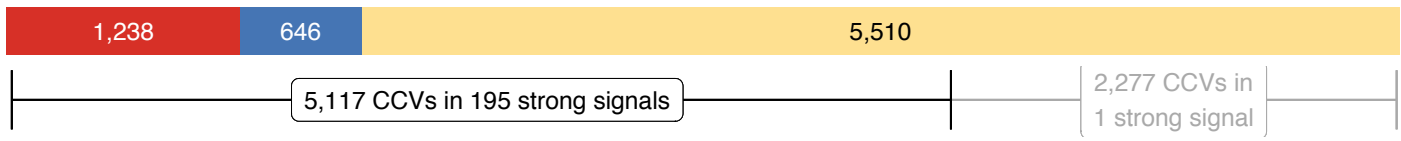

Signal phenotype classification BCAC-CIMBA

66 ER-positive 36 ER-negative 104 ER-neutral

\begin{tabular}{|c|c|c|c|}
\hline 1,238 & $646(226)$ & $5,510(32)$ & \\
\hline & 5,375 CCVs in 205 strong signals & & 2,277 CCVs in \\
\hline
\end{tabular}

PAINTOR Posterior Probability
$\geq 0.9 \geq 0.8-0.9$
$\geq 0.5-0.8$
$\geq 0.3-0.5$

\begin{tabular}{l|l|l|l}
10 & 5 & 35 & 87
\end{tabular}


Figure 2. Determining independent risk signals and credible candidate variants (CCVs). (a) Number of independent signals per region identified through multinomial stepwise logistic regression. (b) Signal classification according to their confidence into strong and moderate confidence signals. (c) Number of CCVs per signal at strong confidence signals identified through multinomial stepwise logistic regression. (d) Number of CCVs per signal at moderate confidence signals identified through multinomial stepwise regression. (e) Subtype classification of strong signals into ER-positive, ER-negative and signals equally associated with both phenotypes (ER-neutral) from BCAC analysis. (f) Subtype classification from the meta-analysis of BCAC and CIMBA. Between brackets, number of CCVs from the meta-analysis of BCAC and CIMBA. (g) Number of variants at different posterior probability thresholds. 15 variants reach a PP $\geq 80 \%$ by at least one of the three models (ER-all, ER-positive, ER-negative).

making 206 strong-evidence signals (17\% ER-negative) containing 7,652 CCVs in total (Figure 2F). More than one quarter of the CCVs $(2,277)$ were accounted for by one signal, resulting from strong linkage disequilibrium with a copy number variant. The remaining analyses focused on the other 205 strong signals across 128 regions (Supplementary Table $2 \mathrm{C}$ ).

CCVs are over-represented in active gene-regulatory regions and transcription factor binding sites (TFBSs)

We constructed a database of mapped genomic-features in seven primary cells derived from normal breast and 19 breast cell lines using publicly available data, resulting in 811 annotation tracks in total. These ranged from general features, such as whether a variant was in an exon or in open chromatin, to more specific features, such a cell-specific TF binding or histone mark (determined through ChIP-Seq experiments) in breast-derived cells or cell lines. Using logistic regression, we examined the overlap of these genomic-features with the positions of 5,117 CCVs in the 195 strong-evidence BCAC signals versus the positions of 622,903 variants excluded as credible candidates in the same regions (Supplementary 
Figure 1A, Supplementary Table 3). We found significant enrichment of CCVs (FDR 5\%) in the following genomic-features:

(i) Open chromatin (determined by DNase-seq and FAIRE-seq) in ER-positive breast cancer cell-lines and normal breast (Figure 3A). Conversely, we found depletion of CCVs within heterochromatin (determined by the H3K9me3 mark in normal breast, and by chromatin-state in ER-positive cells ${ }^{27}$ ).

(ii) Actively transcribed genes in normal breast and ER-positive cell lines (defined by H3K36me3 or H3K79me2 histone marks, Figure 3A). Enrichment was larger for ER-neutral CCVs than for those affecting either ER-positive or ER-negative tumors.

(iii) Gene regulatory regions. CCVs overlapped distal gene regulatory elements in ER-positive breast cancer cells lines (defined by H3K4me1 or H3K27ac marks, Figure 3B). This was confirmed using the ENCODE definition of active enhancers in MCF-7 cells (enhancer-like regions defined by combining DNase and H3K27ac marks), as well as the definition of ${ }^{28}$ and ${ }^{27}$ (Supplementary Table 3). Under these more stringent definitions, enrichment among ER-positive CCVs was significantly larger than ERnegative or ER-neutral CCVs. Data from ${ }^{27}$, showed that $73 \%$ of active enhancer regions overlapped by ER-positive CCVs in ER-positive cells (MCF-7), are inactive in the normal HMEC breast cell line; thus, these enhancers appear to be MCF-7-specific.

We also detected significant enrichment of CCVs in active promoters in ER-positive cells (defined by H3K4me3 marks in T-47D), although the evidence for this effect was weaker than for distal regulatory elements (defined by H3K27ac marks in MCF-7, Figure 3B). Only ER-positive CCVs were significantly 
a
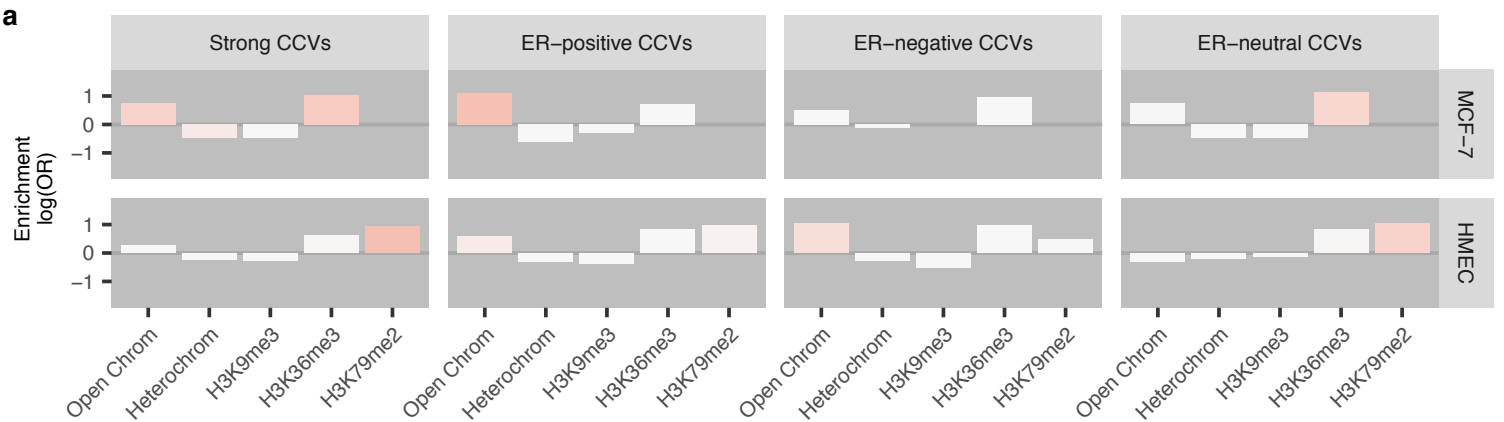

b
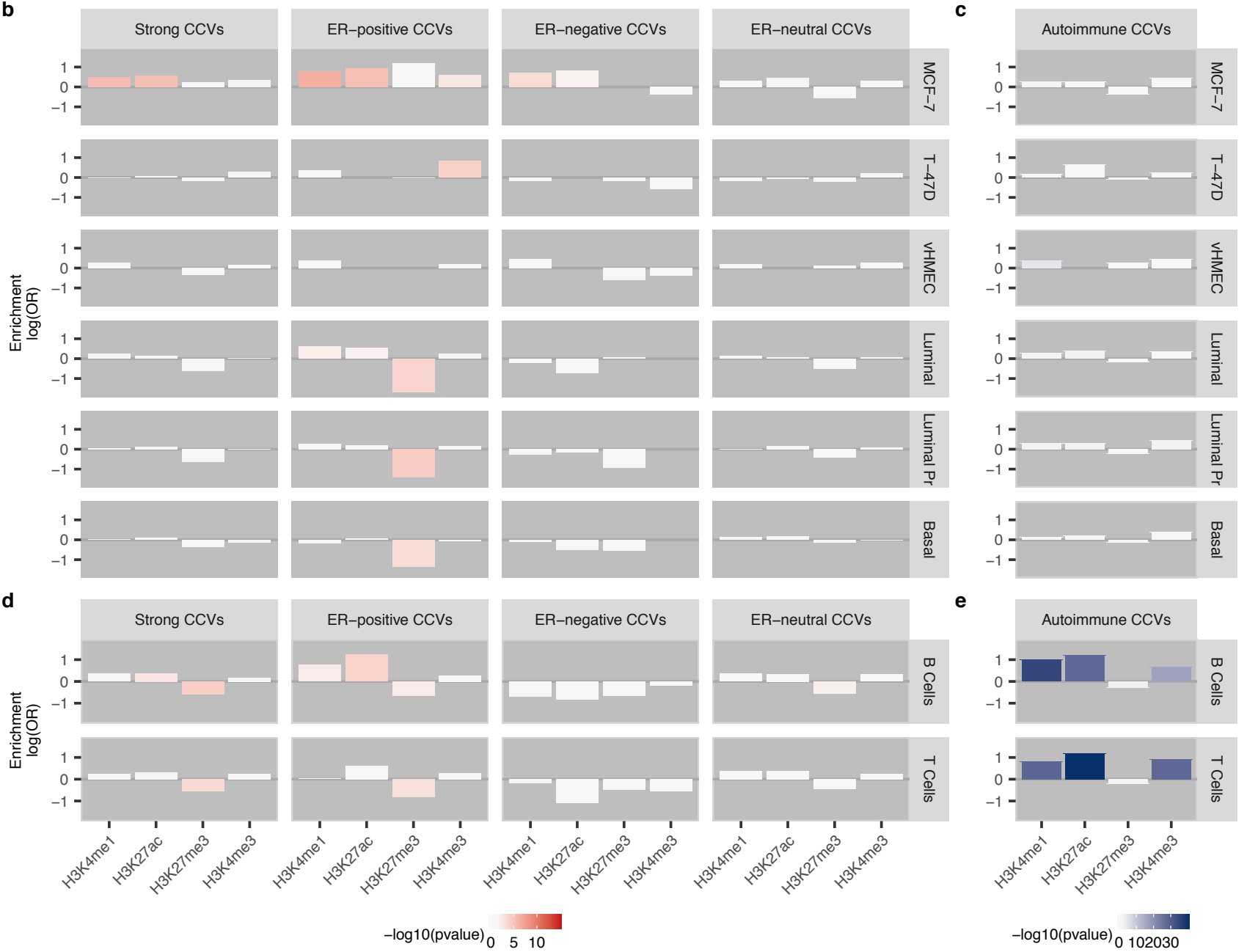

-log10(pvalue) 0102030

ER-positive CCVs

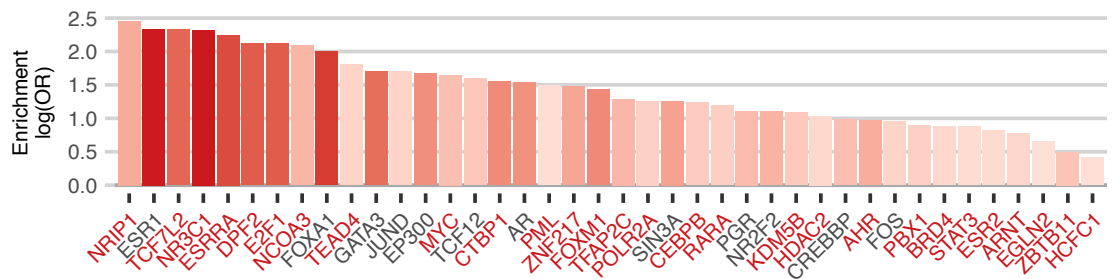

g ER-negative CCVs h ER-neutral CCVs
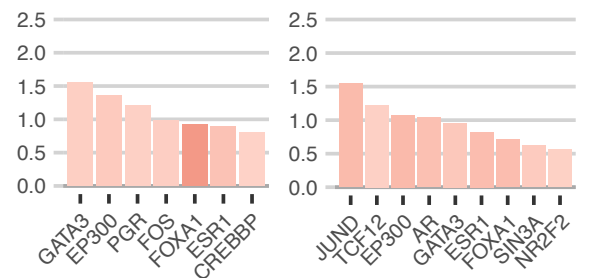
Figure 3. Overlap of CCVs with gene regulatory regions gene bodies and transcription factor binding sites. (a) Breast cancer CCVs overlap with chromatin states and broad breast cells epigenetic marks. (b) Breast cancer CCVs overlap with breast cells epigenetic marks. (c) Autoimmune CCVs overlap with breast cells epigenetic marks. (d) Breast cancer CCVs overlap with autoimmune-related epigenetic marks. (e) Autoimmune CCVs overlap with autoimmune-related epigenetic marks. (f) Significant ERpositive CCVs overlap with transcription factors binding sites. TFBSs found significant for ER-positive CCVs are highlighted in red ( $x$ axis labels). (g) Significant ER-negative CCVs overlap with transcription factors binding sites. (h) Significant ER-neutral CCVs overlap with transcription factors binding sites. Strong column: analysis with all CCVs at strong signals. ER-positive, ER-negative, ER-neutral: analysis of CCVs at strong signals stratified by phenotype.

enriched in T-47D active promoters. Conversely, CCVs were depleted among repressed gene-regulatory elements (defined by H3K27me3 marks) in normal breast (Figure 3B). As a control, we performed similar analyses with autoimmune disease $\mathrm{CCVs}^{29}$ (Methods) and relevant B and T cells (Figures 3B-E). The strongest evidence of enrichment of breast cancer CCVs was found at regulatory regions active in ER-positive cells (Figure 3B), whereas enrichment of autoimmune CCVs was in regulatory regions active in $\mathrm{B}$ and $\mathrm{T}$ cells (Figure $\mathbf{3 E}$ ). We also compared the enrichment of our CCVs in enhancer-like and promoter-like regions (defined by ENCODE; Supplementary Figure 1B). The strongest evidence of enrichment of ER-positive CCVs in enhancer-like regions was found in MCF-7 cells, the only ER-positive cell line in ENCODE (Supplementary Figure 1B). These results highlight both the tissue- and diseasespecificity of these histone marked gene regulatory regions.

(iv) We observed significant enrichment of CCVs in the binding sites for 40 TFBSs determined by ChIPSeq (Figures 3F-H). The majority of the experiments were performed in ER-positive cell lines (90 TFBSs, 20 with data in ER-negative cell lines, 76 in ER-positive cell lines, and 16 in normal breast). These TFBSs 
overlap each other and histone marks of active regulatory regions (Supplementary Figure 2). Enrichment in five TFBSs (ESR1, FOXA1, GATA3, TCF7L2, E2F1) has been previously reported ${ }^{2,30}$. All 40 TFBSs were significantly enriched in ER-positive CCVs (Figure 3F), seven were also enriched in ERnegative CCVs and nine in ER-neutral CCVs (Figures 3G-H). ESR1, FOXA1, GATA3 and EP300 TFBSs were enriched in all CCV ER-subtypes. However, the enrichment for ESR1, FOXA1 or GATA3 was stronger for ER-positive CCVs than for ER-negative or ER-neutral.

\section{CCVs significantly overlap consensus transcription factor binding motifs}

We investigated whether CCVs were also enriched within consensus TF binding motifs by conducting a motif-search within active regulatory regions (ER-positive CCVs at H3K4me1 marks in MCF-7). We identified 30 motifs, from eight TF families, with enrichment in ER-positive CCVs (FDR 10\%, Supplementary Table 4A) and a further five motifs depleted among ER-positive CCVs. To assess whether the motifs appeared more frequently than by chance at active regulatory regions overlapped by our ER-positive CCVs, we compared motif-presence in a set of randomized control sequences (Methods). Thirteen of 30 motifs were more frequent at active regulatory regions with ER-positive CCV enrichment; these included seven homeodomain motifs and two fork head factors (Supplementary Table 4B).

When we looked at the change in predicted binding affinity, 57 ER-positive signals (86\%) included at least one CCV predicted to modify the binding affinity of the enriched TFBSs ( $\geq 2$-fold, Supplementary Table 4C). Forty-eight ER-positive signals (73\%) had at least one CCV predicted to modify the binding 
affinity $>10$-fold. This analysis validates previous reports of breast cancer causal variants that alter DNA binding affinity for FOXA1 3,30

\section{Bayesian fine -mapping incorporating functional annotations and linkage disequilibrium}

As an alternative statistical approach, we applied PAINTOR ${ }^{31}$ to the same 128 regions (Figure 1). In brief, PAINTOR integrates genetic association results, linkage disequilibrium (LD) structure, and enriched genomic features in an empirical Bayes framework and derives the posterior probability (PP) of each variant being causal, conditional on available data. We identified seven variants with high posterior probability (HPP $\geq 80 \%$ ) of being causal for all breast cancer types and ten for the ER-positive subtype (Table 1); two of these had HPP > 80\% for both ER-positive and all breast cancer types. These 15 HPP variants (HPPVs; $\geq 80 \%$ ) were distributed across 13 regions. We also identified an additional 35 variants in 25 regions with HPP ( $\geq 50 \%$ and $<80 \%$ ) for ER-positive, ER-negative, or overall breast cancer (Figure 2G).

Consistent with the CCV analysis, we found evidence that most regions contained multiple HPPVs; the sum of PP across all variants in a region (an estimate of the number of distinct causal variants in the region) was $>2.0$ for $84 / 86$ regions analyzed for overall breast cancer, with a maximum of 16.1 and a mean of 6.4. For ER-positive cancer, $46 / 47$ regions had total PP $>2.0$ (maximum 18.3, mean 6.5) and for ER-negative, 17/23 regions had total PP > 2.0 (maximum 9.1, mean 3.2).

Although for many regions we were not able to identify HPP variants, we were able to reduce the proportion of variants needed to account for $80 \%$ of the total PP in a region to under $5 \%$ for 65 regions 
for overall, 43 for ER-positive, and 18 for ER-negative breast cancer (Figures S3A-C). PAINTOR analyses were also able to reduce the set of likely causal variants in many cases. After summing the PPs for CCVs in each of the overall breast cancer signals, 39/100 strong-evidence signals had a total PP $>1.0$. The number of CCVs in these signals ranged from 1 to 375 (median 24), but the number of variants needed to capture 95\% of the total PP in each signal ranged from 1 to 115 (median 12), representing an average reduction of $43 \%$ in the number of variants needed to capture the signal.

PAINTOR and CCV analyses were generally consistent, yet complementary. Only $3.3 \%$ of variants outside of the set of strong-signal CCVs for overall breast cancer had PP > 1\%, and only $48(0.013 \%)$ of these had PP > 30\% (Supplementary Figure 3D). At ER-positive and ER-negative signals respectively, $3.1 \%$ and $1.6 \%$ of the non-CCVs at strong signals had PP > 1\%, and $40(0.019 \%)$ and $3(0.003 \%)$ of these had PP > 30\% (Figures S3E-F). For the non-CCVs at strong-evidence signals with PP > 30\%, the relatively high PP may be driven by the addition of functional annotation. The incorporation of functional annotation (relative to a PAINTOR model with no functional annotation) more than doubled PP for $64 / 88$ variants.

\section{CCVs co-localize with variants controlling local gene expression}

We used four breast-specific expression quantitative trait loci (eQTL) data sets; to identify variants associated with differences in gene expression (eVariants): tumor tissue from the Nurses' Health Study (NHS) ${ }^{32}$ and The Cancer Genome Atlas (TCGA) ${ }^{33}$, and normal breast tissue from the NHS and the Molecular Taxonomy of Breast Cancer International Consortium (METABRIC) ${ }^{34}$. We then examined the overlap of eVariants (defined as an eVariant within two orders of magnitude of the most significant 
eVariant for a particular gene) with CCVs (Methods). There was significant overlap of CCVs with eVariants from both the NHS normal and breast cancer tissue studies (normal breast OR $=2.70, p$-value $=1.7 \times 10^{-5} ;$ tumor tissue $\mathrm{OR}=2.34, \mathrm{p}$-value $=2.6 \times 10^{-4} ;$ Supplementary Table 3$)$. ER-neutral CCVs overlapped with eVariants in normal tissue more frequently than did ER-positive and ER-negative CCVs $\left(\mathrm{OR}_{\mathrm{ER} \text {-neutral }}=3.51, \mathrm{p}\right.$-value $\left.=1.3 \times 10^{-5}\right)$. Cancer risk CCVs overlapped credible eVariants in 128/205 $(62 \%)$ signals in at least one of the datasets (Tables S5A-B). Sixteen additional variants with PP $\geq 30 \%$, not included among the CCVs, also overlapped with a credible eVariant (Tables S5A-B).

\section{Transcription factors and known somatic breast cancer drivers are overrepresented among prioritized target genes}

We assumed that causal variants function by affecting the behavior of a local target gene. However, it is challenging to define target genes or to determine how they may be affected by the causal variant. Few potentially causal variants directly affect protein coding: we observed 67/5,375 CCVs, and 19/137 HPPVs ( $\geq 30 \%)$ in protein-coding regions. Of these, $33(0.61 \%)$ were predicted to create a missense change, one a frameshift, and another a stop-gain, while 30 were synonymous $(0.59 \%$, Supplementary Table 5C). Four hundred and ninety-nine CCVs at 94 signals, and four additional HPPV ( $\geq 30 \%)$, are predicted to create new splice sites or activate cryptic splice sites in 126 genes (Supplementary Table 5D). These results are consistent with previous observations that majority of common susceptibility variants are regulatory.

We applied an updated version of our pipeline INQUISIT - integrated expression quantitative trait and in-silico prediction of GWAS targets) ${ }^{2}$ to prioritize potential target genes from 5,375 CCVs in strong 
signals and all 138 HPPVs ( $\geq 30 \%$; Supplementary Table 2 C). The pipeline predicted 1,204 target genes from 124/128 genomic regions examined. As a validation we examined the overlap between INQUISIT predictions and 278 established breast cancer driver genes ${ }^{35-39}$. Cancer driver genes were overrepresented among high confidence (Level 1) targets; a 6-fold increase over expected from CCVs and 16-fold from HPPVs; $p$-value $=1 \times 10^{-6}$; Supplementary Figure 4A). Notably, ten cancer driver genes (CCND1, CHEK2, ESR1, FGFR2, GATA3, MAP3K1, MYC, TBX3, XBP1 and ZFP36L1) were predicted from the HPPVs derived from PAINTOR. Cancer driver gene status was consequently included as an additional weighting factor in the INQUISIT pipeline. TF genes ${ }^{40}$ were also enriched amongst high-confidence targets predicted from both CCVs $\left(2\right.$-fold, $\mathrm{p}$-value $\left.=4 \times 10^{-4}\right)$ and HPPVs $\left(3\right.$-fold, $\mathrm{p}$-value $=7 \times 10^{-3}$, Supplementary Figure 4A).

In total INQUISIT prioritized 178 high-confidence target genes (Table 2, Supplementary Table 6). Significantly more genes were targeted by multiple independent signals $(N=165)$ than expected by chance $\left(p\right.$-value $=4.6 \times 10^{-8}$, Supplementary Figure 4B, Figure 4A). Six high-confidence predictions came only from HPPVs, although three of these (IGFBP5, POMGNT1 and WDYHV1) had been predicted at lower confidence from CCVs. Target genes included 20 that were prioritized via potential coding/splicing changes (Supplementary Table 7), nine via promoter variants (Supplementary Table 8), and 166 via distal regulatory variants (Supplementary Table 9). We illustrate genes prioritized via multiple lines of evidence in Figure 4A.

Three examples of INQUISIT using genomic features to identify predict target genes. Based on capture $\mathrm{Hi}-\mathrm{C}$ and ChIA-PET chromatin interaction data, NRIP1 is a predicted target of intergenic CCVs and HPPVs 


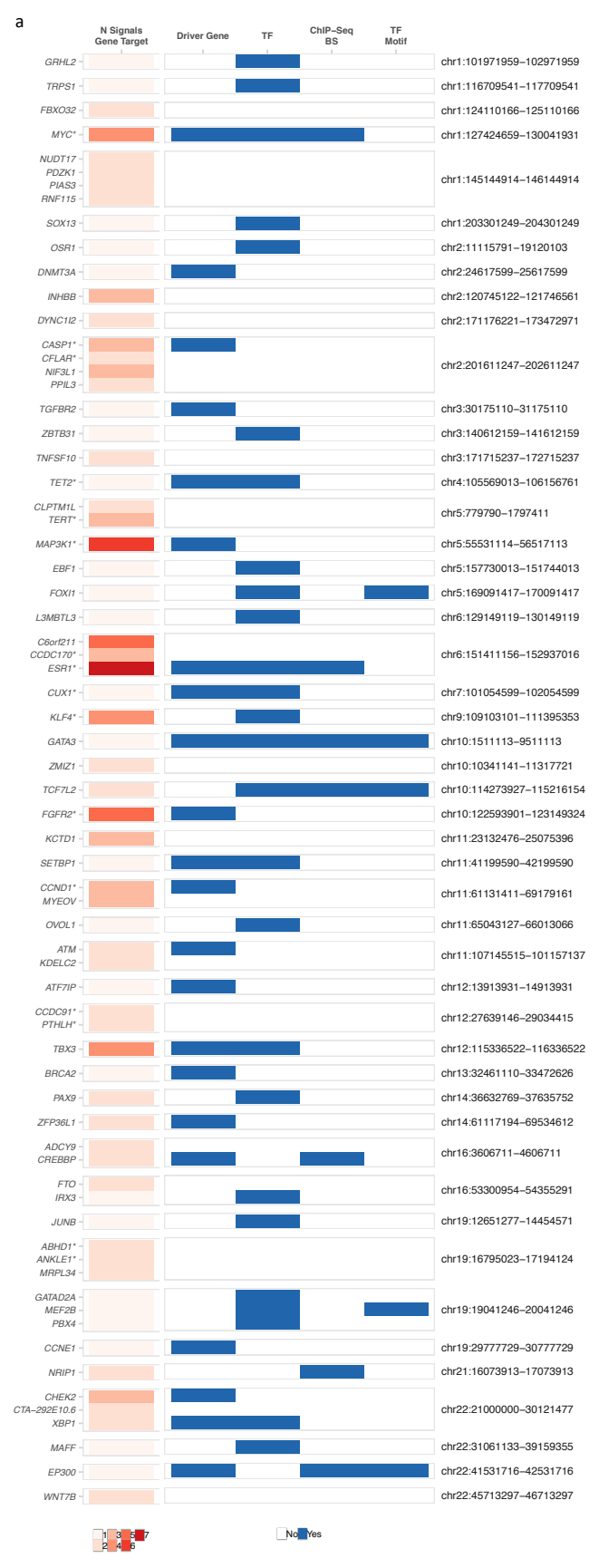

b
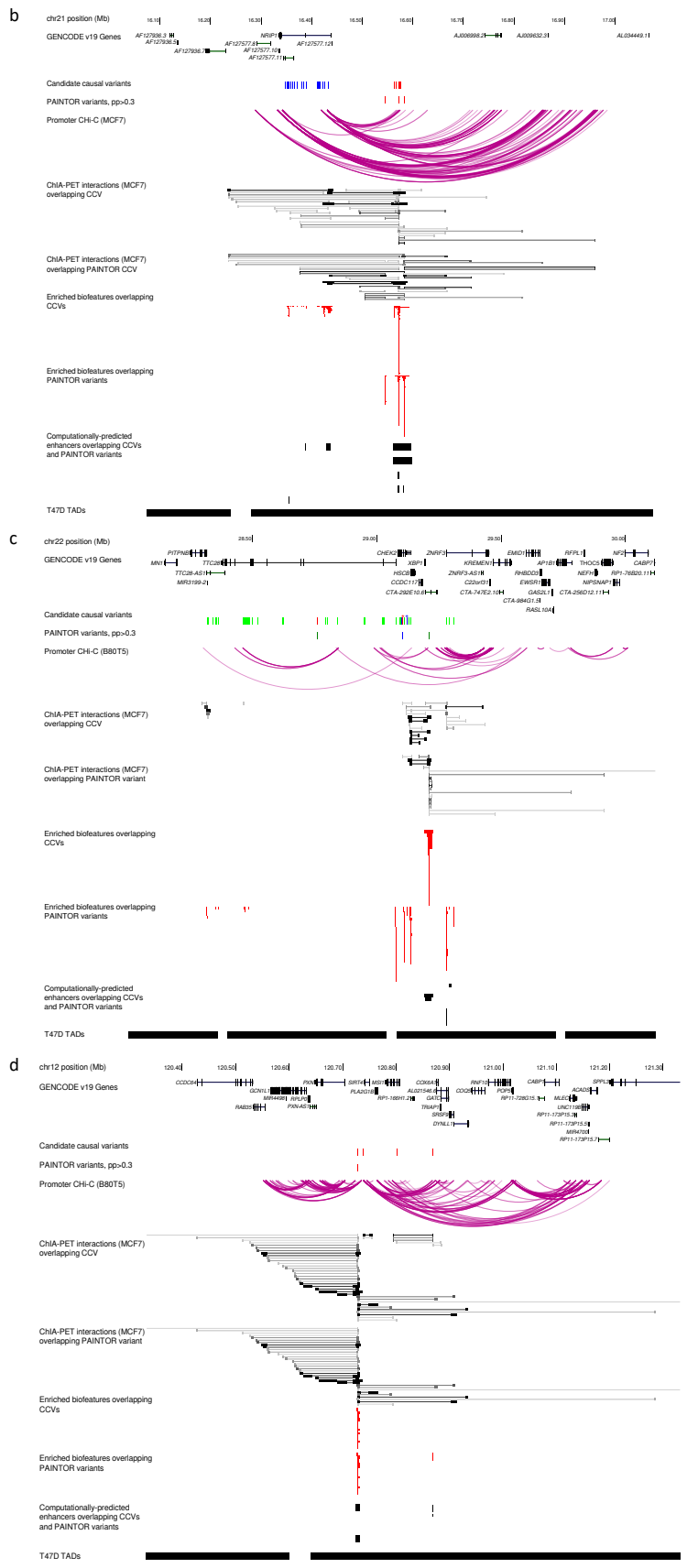

Figure 4. Predicted target genes are enriched in known breast cancer driver genes and transcription factors. (a) 79 target genes that fulfil at least one of the following criteria: are targeted by more than one independent signal, are known driver genes, transcription factor genes, or their binding sites (ChIPSeq BS) or consensus motif (TF Motif) are significantly overlapped by CCVs. *Genes with published functional follow up. (b-d) In each panel, CCVs and PAINTOR variants with posterior probability $>0.3$ are shown, with independent signals in different colors. Chromatin interactions are shown as arcs (Capture $\mathrm{Hi}-\mathrm{C}$ from selected breast cell lines) or boxes connected by lines, colored with gray-scale according to 
interaction score (ENCODE ChIA-PET). Biofeatures which overlap CCVs from the global genomic enrichment analysis are depicted as red boxes. Computationally predicted enhancers including PreSTIGE, FANTOM5 and super-enhancers which overlap risk variants are represented by black boxes. High confidence INQUISIT target gene predictions include NRIP1 (b), CHEK2 and XBP1 (c), and RPLPO and $M S I 1$ (d).

at chr21q21 (Figure 4B). Multiple target genes were predicted at chr22q12, including the driver genes CHEK2 and XBP1 (Figure 4C). A third example at chr12q24.31 is a more complicated scenario with two Level 1 targets: RPLPO ${ }^{41}$ and a modulator of mammary progenitor cell expansion, MSI ${ }^{42}$ (Figure 4D).

Target gene pathways include DNA integrity-checkpoint, apoptosis, developmental processes and the immune system

We performed pathway analysis to identify common processes using INQUSIT high confidence target protein-coding genes (Figure 5A) and identified 457 Gene Ontology (GO) terms and 265 pathways at an FDR of 5\% (Supplementary Table 10). These were grouped into 99 themes by common ancestor GO terms, pathways, or TF classes (Figure 5B). We found that $24 \%(14 / 58)$ of the ER-positive target genes were classified within developmental process pathways (including mammary development), $17 \%$ in immune system and a further $16 \%$ in cell differentiation pathways. Of genes targeted by ER-neutral signals, $19 \%$ (16/85) were classified in developmental process pathways, $18 \%$ in apoptotic process, and a further $16 \%$ in immune system pathways. The top themes of genes targeted by ER-negative signals were DNA integrity checkpoint $(20 \%, 7 / 35)$, immune system, response to stimulus, and apoptotic processes, each containing $17 \%(6 / 35)$ of the genes. 
a

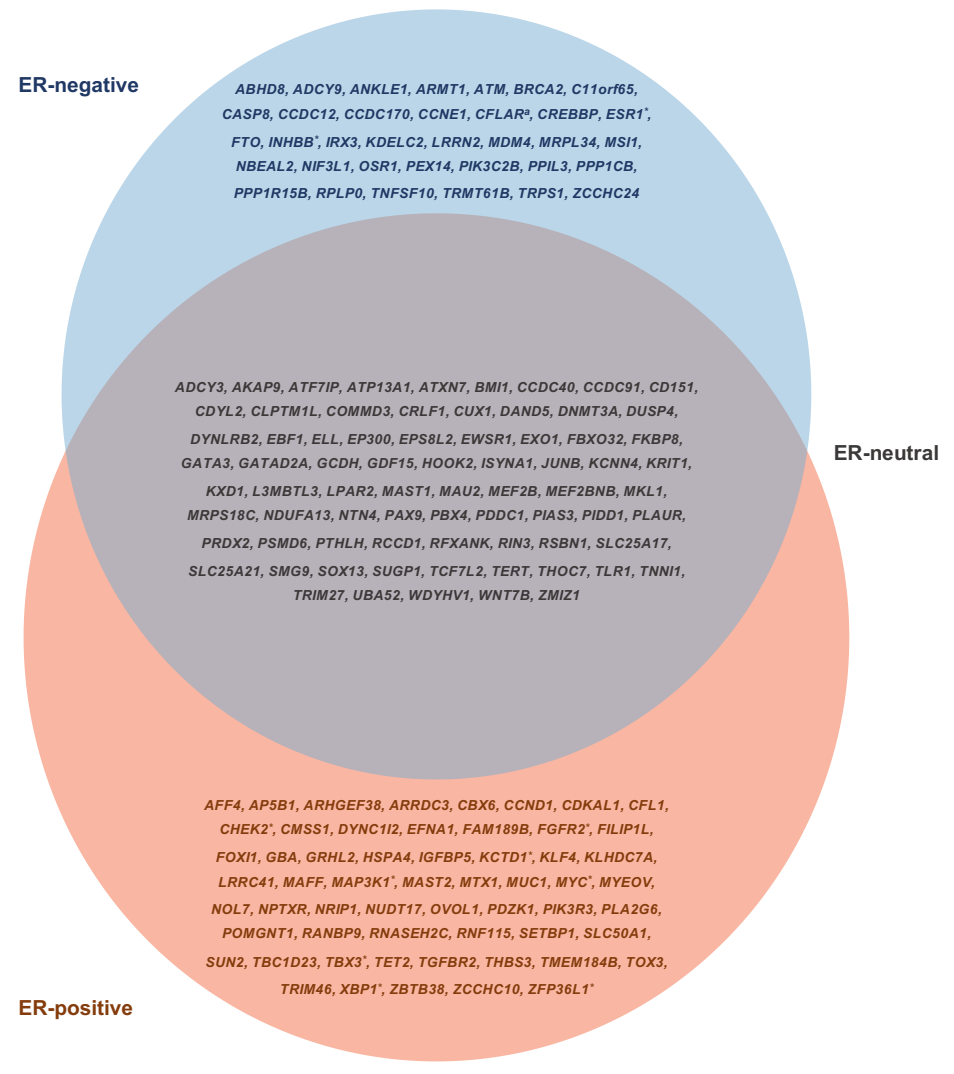

b
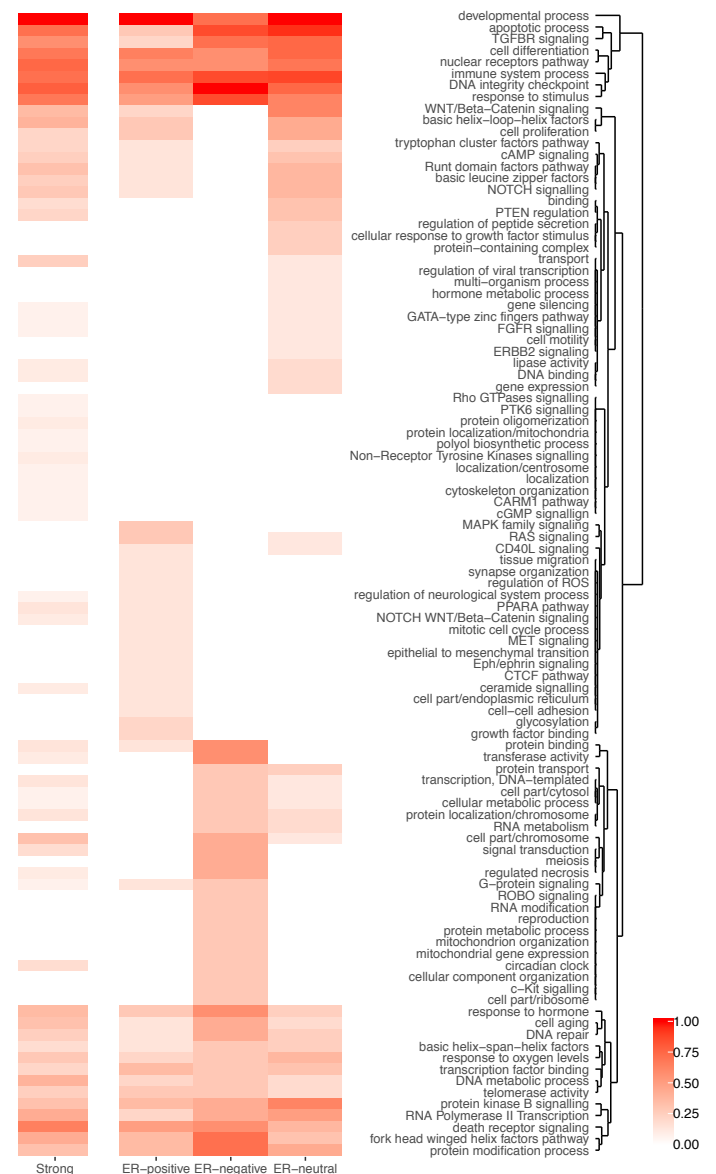

Figure 5. Predicted target genes by phenotype and significantly enriched pathways. (a) Venn diagram showing the associated phenotype (ER-positive, ER-negative, ER-neutral) for the Level 1 target genes, predicted by the CCVs and HPPVs. * ER-positive or ER-negative target genes also targeted by ER-neutral signals. (b) Heatmap showing clustering of pathway themes over-represented by INQUISIT Level 1 target genes. Color represents the relative number of genes per phenotype within enriched pathways, grouped by common themes. ER-positive, ER-negative, ER-neutral, and all phenotypes together (strong).

Novel pathways revealed by this study include TNF-related apoptosis-inducing ligand (TRAIL) signaling, the AP-2 transcription factors pathway, and regulation of I $\mathrm{KB}$ kinase/NF- $\kappa B$ signaling. Of note, the latter of these is specifically overrepresented among ER-negative target genes. We also found significant 
overrepresentation of additional carcinogenesis-linked pathways including CAMP, NOTCH, PI3K, RAS, WNT/Beta-catenin, and of receptor tyrosine kinases signaling, including FGFR, ERBB, or TGFBR ${ }^{43-47}$. Also of note, our target genes are significantly overrepresented in DNA damage checkpoint, DNA repair pathways, as well as programmed cell death pathways, such as apoptotic process, regulated necrosis, and death receptor signaling-related pathways.

\section{DISCUSSION}

We have performed multiple, complementary analyses on 150 breast cancer associated regions, originally found by GWAS, and identified 362 independent risk signals, 205 of these with high confidence ( $p$-value $<10^{-6}$ ). We observed most regions contain multiple independent signals, the greatest number (nine) in the region surrounding ESR1 and its co-regulated genes, and on 2q35, where IGFBP5 appears to be a key target. We have used two complementary approaches to identify likely causal variants within each region: a Bayesian approach, PAINTOR, which integrated genetic associations, LD and informative genomic features, confirmed most associations found by the more traditional, multinomial regression approach, and also identified additional variants. Specifically, the Bayesian method highlighted 15 variants that are highly likely to be causal (HPP $\geq 80 \%$ ). From these approaches we have identified a single variant, likely to be causal, at each of 34/205 signals (Table 1). Of these, only rs16991615 (MCM8 p.E341K) and rs7153397 (CCDC88C, a cryptic splice-donor site) were predicted to affect protein-coding sequences. However, in other signals we also identified four coding changes previously recognized as deleterious, including the stop-gain rs11571833 (BRCA2 p.K3326*, Meeks et al., 2016) ${ }^{48}$ and two CHEK2 coding variants; the frameshift rs555607708 ${ }^{49,50}$, and a missense variant, rs17879961 ${ }^{51,52}$. In addition, a splicing variant, rs10069690, in TERT results in the truncated 
protein INS1b ${ }^{19}$, decreased telomerase activity, telomere shortening, and increased DNA damage response ${ }^{53}$

Having identified potential causal variants within each signal, we aimed to uncover their functions at the DNA level and as well as trying to predict their target gene(s). Looking across all 150 regions, a notable feature is that many likely causal variants implicated in ER-positive cancer risk, lie in generegulatory regions marked as open and active in ER-positive breast cells, but not in other cell types. Moreover, a significant proportion of potential causal variants overlap the binding sites for transcription factor proteins ( $n=40$ from ChIP-Seq) and co-regulators ( $n=64$ with addition of computationally derived motifs). Furthermore, nine proteins also appear in the list of high-confidence target genes, hence the following genes and their products have been implicated by two different approaches: CREBBP, EP300, ESR1, FOXI1, GATA3, MEF2B, MYC, NRIP1 and TCF7L2. Most of these proteins already have established roles in estrogen signaling. CREBBP, EP300, ESR1, GATA3, and MYC are also known cancer driver genes that are frequently somatically mutated in breast tumors.

In contrast to ER-positive signals, we identified fewer genomic features enriched in ER-negative signals. This may reflect the common molecular mechanisms underlying their development, but the power of this study was limited, despite including as many patients with ER-negative tumors as possible from the BCAC and CIMBA consortia. Less than $20 \%$ of genomic signals confer a greater risk of ER-negative cancer and there is little publicly available ChIP-Seq data on ER-negative breast cancer cell lines. The heterogeneity of ER-negative tumors may also have limited our power. Nevertheless, we have identified 35 target genes for ER-negative likely causal variants. Some of these already had functional 
evidence supporting their role: including CASP8 ${ }^{54}$ and $M D M 4^{55}$. Most targets, however, currently have no reported function in ER-negative breast cancer development.

Finally, we examined the gene-ontology pathways in which target genes most often lie. Of note, $12 \%$ (20/167) of all high-confidence target genes and $17 \%$ of ER-negative target predictions are in immune system pathways. Among the significantly enriched pathways were $\mathrm{T}$ cell activation, interleukin

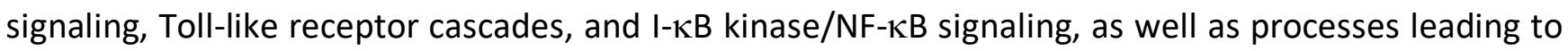
activation and perpetuation of the innate immune system. The link between immunity, inflammation and tumorigenesis has been extensively studied ${ }^{56}$, although not primarily in the context of susceptibility. Four ER-negative high confidence target genes (CASP8, CFLAR, ESR1, TNFSF10) lie in the

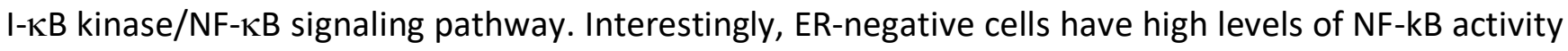
when compared to ER-positive ${ }^{57}$. A recent expression-methylation analysis on breast cancer tumor tissue also identified clusters of genes correlated with DNA methylation levels, one enriched in ER signaling genes, and a second in immune pathway genes ${ }^{58}$.

These analyses provide strong evidence for more than 200 independent breast cancer risk signals, identify the plausible cancer variants and define likely target genes for the majority of these. However, notwithstanding the enrichment of certain pathways and transcription factors, the biological basis underlying most of these signals remains poorly understood. Our analyses provide a rational basis for such future studies into the biology underlying breast cancer susceptibility. 


\section{METHODS}

\section{Study samples}

Epidemiological data for European women were obtained from 75 breast cancer case-control studies participating in the Breast Cancer Association Consortium (BCAC) (cases: 40,285 iCOGS, 69,615 OncoArray; cases with ER status available: 29,561 iCOGS, 55,081 OncoArray); controls: 38,058 iCOGS, 50,879 OncoArray). Details of the participating studies, genotyping calling and quality control are given in 2,22,23, respectively. Epidemiological data for BRCA1 mutation carriers were obtained from 60 studies providing data to the Consortium of Investigators of Modifiers of BRCA1 and BRCA2 (CIMBA) (affected 1,591 iCOGS, 7,772 OncoArray; unaffected 1,665 iCOGS, 7,780 OncoArray). This dataset has been described in detail previously ${ }^{1,59,60}$. All studies provided samples of European ancestry. Any nonEuropean samples were excluded from analyses.

\section{Variant selection, genotyping and imputation}

A dense set of variants surrounding susceptibility variants known at the time of design was selected for genotyping on the iCOGS array. To select markers for comprehensive interrogation of these denselygenotyped regions, all known variants from the March 2010 release of the 1000 Genomes Project with MAF $>0.02$ in Europeans were identified, and all variants that were correlated with the published GWAS variants at $r 2>0.1$ together with a set of variants designed to tag all remaining variants at $r 2>0.9$ were selected. Twenty-one regions were densely genotyped with iCOGS (http://ccge.medschl.cam.ac.uk/files/2014/03/iCOGS_detailed_lists_ALL1.pdf). In total 26,978 iCOGS genotyped variants across the 152 mapped regions passed QC criteria. Similarly, all known associated risk variants at the time of the design of the OncoArray were included in the genotyping array. Variants 
for fine-mapping were selected defining a $1 \mathrm{Mb}$ interval surrounding a known hit. Overlapping regions were merged into a single interval including $500 \mathrm{~kb}$ either side of each lead signal. From among designable variants, all variants correlated with the known hits at $r^{2}>0.6$, all variants from lists of potentially functional variants, defined through RegulomeDB, and a set of variants designed to tag all remaining variants at $r^{2}>0.9$ were selected. Seventy-three regions were densely genotyped with OncoArray. In total 58,339 OncoArray genotyped variants across the 152 mapped regions passed QC criteria.

We imputed genotypes for the remaining variants at the genomic regions defined in the OncoArray design using IMPUTE2 ${ }^{61}$, and the October 2014 release of the 1000 Genomes Project as a reference. To improve the accuracy at low frequency variants we used the standard IMPUTE2 MCMC algorithm for follow-up imputation (more detailed description of the parameters used can be found in ${ }^{21}$ )We genotyped or successfully imputed 639,118 variants all having imputation info score $\geq 0.3$ and minor allele frequencies $(\mathrm{MAF}) \geq 0.001$ in both the iCOGS and OncoArray datasets. Imputation summaries, and coverage for each of the analyzed regions stratified by allele frequency can be found in

\section{Supplementary Table 1B.}

\section{BCAC Statistical analyses}

Per-allele odds ratios (OR) and standard errors (SE) were estimated for each variant using logistic regression. We ran this analysis separately for iCOGS and OncoArray, and separately for overall, ERpositive and ER-negative breast cancer. The association between each variant and breast cancer risk was adjusted by study (iCOGS) or country (OncoArray), and eight (iCOGS) or ten (OncoArray) ancestry- 
informative principal components. The statistical significance for each variant was derived using a Wald test.

\section{Defining the significance threshold}

To establish an appropriate significance threshold for independent signals, all variants evaluated in the meta-analysis were included in logistic forward selection regression analyses for overall breast cancer risk in iCOGS cohort, run independently for each of the regions. We evaluated five $p$-value thresholds for inclusion: $<1 \times 10^{-4},<1 \times 10^{-5},<1 \times 10^{-6},<1 \times 10^{-7}$, and $<1 \times 10^{-8}$. The most parsimonious iCOGS models were tested in OncoArray, and the false discovery rate (FDR) at $1 \%$ level for each one of the thresholds was estimated using the Benjamini-Hochberg procedure ${ }^{62}$. At a $1 \%$ FDR threshold, $72 \%$ of variants of variants significant at $p<10^{-4}$ replicated on the OncoArray, whereas $94 \%$ of variants significant at $p<10^{-}$

${ }^{6}$ replicated. Based on these analyses, two categories were defined: strong-evidence signals (conditional $p$-values $<10^{-6}$ when the signal is included in the final model fit using the inclusion threshold $p<10^{-4}$ ), and moderate-evidence signals (conditional $p$-values $<10^{-4}$ and $\geq 10^{-6}$ in the final model).

\section{Identification of independent signals}

To identify independent signals, we ran multinomial stepwise regression analyses, separately in iCOGS and OncoArray, for all the variants displaying evidence of association $\left(\mathrm{N}_{\text {variants }}=202,749\right)$. We selected two sets of well imputed variants (imputation info score $\geq 0.3$ in both iCOGS and OncoArray): (a) common and low frequency variants (MAF $\geq 0.01$ ) with a threshold for inclusion of logistic regression $p$-value $\leq 0.05$ in either the iCOGS or OncoArray datasets for at least one of the three phenotypes: overall, ER-positive and ER-negative breast cancer; and (b) rarer variants (MAF $\geq 0.001$ and $<0.01$ ), with a threshold for inclusion of a logistic regression $p$-value $\leq 0.0001$. The same parameters used for adjustment in logistic regression were used in the multinomial regression analysis ( $R$ function 
multinom). The multinomial regression estimates were combined using a fixed-effects meta-analysis weighted by the inverse variance ${ }^{63}$. Variants with the lowest conditional p-value from the meta-analysis of both European cohorts at each step were included into the multinomial regression model. However, if the new variant to be included in the model caused collinearity problems due to be highly correlated with an already selected variant, or showed high heterogeneity ( $p$-value $<10^{-4}$ ) between iCOGS and OncoArray after being conditioned by the variant(s) in the model; we dropped the new variant and repeated this process.

The main signal at 105 of 152 evaluated regions demonstrated genome-wide significance, while 44 were marginally significant $\left(9.89 \times 10^{-5} \geq p\right.$-value $\left.>5 \times 10^{-8}\right)$. For two regions there were no variants significant at $\mathrm{p}<10^{-4}$ (chr14:104712261-105712261; rs10623258 multinomial regression $\mathrm{p}$-value = 2.32 $\times 10^{-4}$; chr19:10923703-11923703, rs322144, multinomial regression $\mathrm{p}$-value $=3.90 \times 10^{-3}$ ). There are four main differences in the dataset used in the analysis and that used in the previous paper that account for these differences : (i) our previous paper ${ }^{2}$ included data from 11 additional GWAS studies $(14,910$ cases and 17,588 controls $)$ that have not been included in the present analysis in order to minimize differences in array coverage; (ii) the present analysis evaluated the risk of ER-positive and ER-negative disease concurrently, whereas in our previous paper the outcome was overall breast cancer risk. ER status was available for only $73 \%$ of the iCOGS and $79 \%$ of the OncoArray breast cancer cases (iii) for the set of samples genotyped with both arrays, ${ }^{2}$ included iCOGS genotypes, while this study includes OncoArray genotypes to maximize the number of samples genotyped with a larger coverage; and (iv) imputation parameters at fine mapping-regions were modified to improve the imputation accuracy of less frequent variants. 
Selection of a credible set of variants candidates to be causal

Variants with p-values within two orders of magnitude of the top variant for each signal, after adjusting for the index variant of preceding signals, were considered as the credible set of candidates to drive the association at each independent signal (CCVs) ${ }^{24} \mathrm{We}$ defined the best model, among those including one CCVs for each signal, by iteratively conditioning on the selected CCVs in the other signals. For ten fine mapping regions (chr4:175328036-176346426, chr5:55531884-56587883, chr6:151418856152937016, chr8:75730301-76917937, chr10:80341148-81387721, chr10:122593901-123849324, chr12:115336522-116336522, chr14:36632769-37635752, chr16:3606788-4606788, chr22:3806883339859355) a model including the lowest conditioned p-value variants (index variant) at each signal could not be found. In that case we estimated the best model as the model with the largest chi-square, from all the possible combinations of credible variants. Variants in strong signals were conditioned by the additional strong confidence signals whereas variants for moderate confidence signals were conditioned by all the independent signals. We then redefined the credible set of candidate variants for each signal using the conditional $p$-values by the additional independent signals. Thus, variants with $p$-values within two orders of magnitude of the top variant for each signal, after adjusting for all the index variant of the additional signals at the locus, were considered as the final credible set of candidates.

\section{Case -only analysis}

Differences in the effect size between ER-positive and ER-negative disease for each index independent variant were assessed using a case-only analysis. We performed logistic regression with ER status as the 
dependent variable, and the lead variant at each strong signal in the fine mapping region as the independent variables. We use FDR (5\%) to adjust for multiple testing.

\section{OncoArray-only stepwise analysis}

To evaluate whether the lower coverage in iCOGS could affect the identification of independent signals, we ran stepwise multinomial regression using only the OncoArray dataset. We identified 249 independent signals. Ninety-two signals, in 67 fine mapping regions, achieved a genome-wide significance level (conditional p-value $<5 \times 10-8$ ). Two hundred and five of these signals were also identified in the meta-analysis with iCOGS. Nine independent variants across ten regions were not evaluated in the combined analysis due to their low imputation info score in iCOGS. Out of these nine signals, two signals would be classified as main primary signals, rs114709821 at region chr1:145144984146144984 (OncoArray imputation info score $=0.72$ ), and rs540848673 at region chr1:149406413150420734 (OncoArray imputation info score $=0.33$ ). Given the low number of additional signals identified in the OncoArray dataset alone, all analyses were based on the combined iCOGS/OncoArray dataset.

\section{CIMBA statistical analysis}

CIMBA provided data from 60 retrospective cohort studies consisting of 9,445 unaffected and 9,363 affected female BRCA1 mutation carriers of European ancestry. Unconditional (i.e. single variant) analyses were performed using a score test based on the retrospective likelihood of observing the genotype conditional on the disease phenotype ${ }^{64,65}$. Conditional analyses, where more than one variant is analyzed simultaneously, cannot be performed in this score test framework. Therefore, 
conditional analyses were performed by Cox regression, allowing for adjustment of the conditionally independent variants identified by the BCAC/DRIVE analyses. All models were stratified by country and birth cohort, and adjusted for relatedness (unconditional models used kinship adjusted standard errors based on the estimated kinship matrix; conditional models used cluster robust standard errors based on phenotypic family data).

Data from the iCOGS array and the OncoArray were analyzed separately and combined to give an overall $B R C A 1$ association by fixed-effects meta-analysis. Variants were excluded from further analyses if they exhibited evidence of heterogeneity (Heterogeneity p-value $<1 \times 10-4$ ) between iCOGS and OncoArray, had MAF $<0.005$, were poorly imputed (imputation info score $<0.3$ ) or were imputed to iCOGS only (i.e. must have been imputed to OncoArray or iCOGS and OncoArray).

\section{Meta-analysis of ER-negative cases in BCAC with BRCA1 mutation carriers from CIMBA}

$B R C A 1$ mutation carrier association results were combined with the BCAC multinomial regression ERnegative association results in a fixed-effects meta-analysis. Variants considered for analysis must have passed all prior $\mathrm{QC}$ steps and have had $\mathrm{MAF} \geq 0.005$. All meta-analyses were performed using the METAL

software ${ }^{66}$.Instances where spurious associations might occur were investigated by assessing the LD between a possible spurious association and the conditionally independent variants. High LD between a variant and a conditionally independent variant within its region causes model instability through collinearity and the convergence of the model likelihood maximization may not reliable. Where the association appeared to be driven by collinearity, the signals were excluded. 


\section{eQTL analysis}

Total RNA was extracted from normal breast tissue in formalin-fixed paraffin embedded breast cancer tissue blocks from 264 Nurses' Health Study (NHS) participants ${ }^{32}$. Transcript expression levels were measured using the Glue Grant Human Transcriptome Array version 3.0 at the Molecular Biology Core Facilities, Dana-Farber Cancer Institute. Gene expression was normalized and summarized into $\log _{2}$ values using RMA (Affymetrix Power Tools v1.18.012); quality control was performed using GlueQC and arrayQualityMetrics v3.24.014. Genome-wide data on variants were generated using the Illumina HumanHap 550 BeadChip as part of the Cancer Genetic Markers of Susceptibility initiative ${ }^{67}$. Imputation to the 1000KGP Phase 3 v5 ALL reference panel was performed using MACH to pre-phase measured genotypes and minimac to impute.

Expression analyses were performed using data from The Cancer Genome Atlas (TCGA) and Molecular Taxonomy of Breast Cancer International Consortium (METABRIC) projects ${ }^{34,38}$. The TCGA eQTL analysis was based on 458 breast tumors that had matched gene expression, copy number and methylation profiles together with the corresponding germline genotypes available. All 458 individuals were of European ancestry as ascertained using the genotype data and the Local Ancestry in admixed Populations (LAMP) software package (LAMP estimate cut-off $>95 \%$ European) ${ }^{68}$. Germline genotypes were imputed into the 1000 Genomes Project reference panel (October 2014 release) using IMPUTE version ${ }^{69,70}$. Gene expression had been measured on the Illumina HiSeq 2000 RNA-Seq platform (gene-level RSEM normalized counts ${ }^{71}$ ), copy-number estimates were derived from the Affymetrix SNP 6.0 (somatic copy-number alteration minus germline copy-number variation called using the GISTIC2 algorithm ${ }^{72}$ ), and methylation beta values measured on the Illumina Infinium HumanMethylation450. 
Expression QTL analysis focused on all variants within each of the 152 genomic intervals that had been subjected to fine-mapping for their association with breast cancer susceptibility. Each of these variants was evaluated for its association with the expression of every gene within $2 \mathrm{Mb}$ that had been profiled for each of the three data types. The effects of tumor copy number and methylation on gene expression were first regressed out using a method described previously ${ }^{73}$. eQTL analysis was performed by linear regression, with residual gene expression as outcome, germline SNP genotype dosage as the covariate of interest and ESR1 expression and age as additional covariates, using the R package Matrix eQTL ${ }^{74}$.

The METABRIC eQTL analysis was based on 138 normal breast tissue samples resected from breast cancer patients of European ancestry. Germline genotyping for the METABRIC study was also done on the Affymetrix SNP 6.0 array, and gene expression in the METABRIC study was measured using the Illumina HT12 microarray platform (probe-level estimates). No adjustment was implemented for somatic copy number and methylation status since we were evaluating eQTLs in normal breast tissue. All other steps were identical to the TCGA eQTL analysis described above.

\section{Genomic feature enrichment}

We explored the overlap of candidate and excluded variants with 90 transcription factors, 10 histone marks, and DNase hypersensitivity sites in in 15 breast cell lines, and eight normal human breast tissues. We analysed data from the Encyclopedia of DNA Elements (ENCODE) Project ${ }^{75,76}$, Roadmap Epigenomics Projects ${ }^{77}$, the International Human Epigenome Consortium ${ }^{78}, 27,79$, The Cancer Genome Atlas (TCGA) ${ }^{33}$, the Molecular Taxonomy of Breast Cancer International Consortium (METABRIC) ${ }^{34}$, ReMap database (We included 241 TF annotations from ReMap (of 2825 total) which 
showed at least $2 \%$ overlap for any of the phenotype SNP sets) ${ }^{80}$, and other data obtained through the National Center for Biotechnology Information (NCBI) Gene Expression Omnibus (GEO). Promoters were defined following the procedure defined in Pellacani et al. ${ }^{79}$, that is $+/-2 \mathrm{~Kb}$ from a gene transcription start site, using an updated version of the RefSeq genes (refGene, version updated 2017-04-11) $)^{81}$. Transcribed regions were defined using the same version of refSeq genes. IncRNA annotation was obtained from Gencode (v19) ${ }^{82}$

To include eQTL results in the enrichment analysis we (i) identified all the genes for which summary statistics were available, for each study; (ii) defined the most significant eQTL variant for each gene (index eQTL variant, $p$-value threshold $\leq 5 \times 10-4$ ); (iii) classified variants with $p$-values within two orders of magnitude of the index eVariant as the credible set of eQTL variants candidate to drive the expression of the gene. Those variants part of at least one eQTL credible set were defined as eVariants. We evaluated the overlap between eQTL credible sets and CCVs (risk variants credible set). We evaluated the enrichment of CCVs for genomic feature using logistic regression, with CCV (vs non-CCV variants) being the outcome. To adjust for the correlation among variants in the same fine mapping region, we used robust variance estimation for clustered observations ( $\mathrm{R}$ function multiwaycov). The associated variants at FDR 5\% were included into a stepwise forward logistic regression procedure to select the most parsimonious model. A likelihood ratio test was used to compare multinomial logistic regression models with and without equality effect constraints to evaluate whether there was heterogeneity among the effect sizes for ER-positive, ER-negative or signals equally associated with both phenotypes (ER-neutral). 
To validate the disease specificity of the regulatory regions identified through this analysis we follow the same approach for the autoimmune related CCVs from ${ }^{29}(\mathrm{~N}=4,192)$. Variants excluded as candidate causal variants, and within $500 \mathrm{~kb}$ upstream and downstream of the index variant for each signal were classified as excluded variants $(N=1,686,484)$. We then tested the enrichment for both the breast cancer and autoimmune CCVs with breast and T and B cell enhancers. We also evaluated the overlap of our CCVs with ENCODE enhancer-like and promoter-like regions for 111 tissues, primary cells, immortalized cell line, and in vitro differentiated cells. Of these, 73 had available data for both enhancer- and promoter-like regions.

\section{Transcription binding site motif analysis}

We conducted a search to find motif occurrences for the transcription factors significantly enriched in the genomic featured. For this we used two publicly available databases, Factorbook ${ }^{83}$ and JASPAR $2016^{84}$. For the search using Factorbook we included the motifs for the transcription factors discovered in the cell lines where a significant enrichment was found in our genomic features analysis. We also searched for all the available motifs for Homo sapiens at the JASPAR database (JASPAR CORE 2016, TFBSTools ${ }^{85}$ )Using as reference the USCS sequence (BSgenome.Hsapiens. USCS.hg19) we created fasta sequences with the reference and alternative alleles for all the variants included in our analysis plus 20 bp flanking each variant. We used FIMO (version 4.11.2, Grant et al., 2011) ${ }^{86}$ to scan all the fasta sequences searching for the JASPAR and Factorbook motifs to identify any overlap of any of the alleles for each of the variants (setting the $\mathrm{p}$-value threshold to $10^{-3}$ ). We subsequently determined whether our CCVs were more frequency overlapping a particular TF binding motif when compared with the excluded variants. We ran these analyses for all the strong signals, but also strong signals stratified by 
ER status. Also, we subset this analysis to the variants located at regulatory regions in an ER-positive cell line (MCF-7 marked by H3K4me1, ENCODE id: ENCFF674BKS) and evaluated whether the ERpositive CCVs overlap any of the motifs more frequently that the excluded variants. We also evaluated the change in total binding affinity caused by the ER-positive CCCR alternative allele for all but one (2:217955891:T:<CN0>:0) of the ER-positive CCVs (MatrixRider ${ }^{87}$ ).

Subsequently, we evaluated whether the MCF-7 regions demarked by H3K4me1 (ENCODE id: ENCFF674BKS), and overlapped by ER-positive CCVs, were enriched in known TFBS motifs. We first subset the ENCODE bed file ENCFF674BKS to identify MCF-7 H3K4me1 peaks overlapped by the ERpositive CCVs $(N=107)$, as well as peaks only overlapped by excluded variants $(N=11,099)$, using BEDTools ${ }^{88}$. We created fasta format sequences using genomic coordinate data from the intersected bed files. In order to create a control sequence set, we used the script included with the MEME Suite (fasta-shuffle-letters) to created 10 shuffled copies of each sequence overlapped by ER-positive CCVs $(N=1,070)$. We then used $\mathrm{AME}^{89}$ to interrogate whether the $107 \mathrm{MCF}-7 \mathrm{H} 3 \mathrm{~K} 4 \mathrm{me} 1$ genomic regions overlapped by ER-positive CCVs were enriched in know TFBS consensus motifs when compared to the shuffled control sequences, or to the MCF-7 H3K4me1 genomic regions overlapped only by excluded variants. We used the command line version of AME (version 4.12.0) selecting as scoring method the total number of positions in the sequence whose motif score $p$-value is less than $10^{-3}$, and using a onetailed Fisher's Exact test as the association test.

\section{PAINTOR analysis}


To further refine the set of CCVs, we performed empirical Bayes fine-mapping using PAINTOR to integrate marginal genetic association summary statistics, linkage disequilibrium patterns, and biological features ${ }^{31,90}$. PAINTOR places higher prior probability for causality on variants that fall in particular genomic features and learns the priors for different genomic features from the aggregate association results across all of the regions being mapped. PAINTOR does not assume a fixed number of causal variants in each region, although it implicitly penalizes non-parsimonious causal models. We applied PAINTOR separately to association results for overall breast cancer (in 85 regions determined to have at least one ER-neutral association or ER-positive and ER-negative association), ER-positive breast cancer (in 48 regions determined to have at least one ER-positive-specific association), and ERnegative breast cancer (in 22 regions determined to have at least one ER-negative-specific association). To avoid artifacts due to mis-matches between the LD in study samples and the LD matrix supplied to PAINTOR, we used association logistic regression summary statistics from OncoArray data only and estimated the LD structure in the OncoArray sample. For each endpoint we fit four models with increasing numbers of genomic features selected from the stepwise enrichment analyses described above: Model 0 (with no genomic features-assumes each variant is equally likely to be causal a priori), Model 1 (with those genomic features selected with stopping rule $p<0.001$ ); Model 2 (with those genomic features selected with stopping rule $p<0.01$ ); and Model 3 (with those genomic features selected with stopping rule $\mathrm{p}<0.05)$.

We used the Bayesian Information Criterion (BIC) to choose the best-fitting model for each outcome. As PAINTOR estimates the marginal log likelihood of the observed Z scores using Gibbs sampling, we used a shrunk mean BIC across multiple Gibbs chains to account for the stochasticity in the log- 
likelihood estimates. We ran PAINTOR four times to generate four independent Gibbs chains estimated

the BIC difference between model $i$ and model $j$ as $\Delta_{i j}=\left(\frac{100}{V+100}\right)\left(B I ́ C_{l}-B I ́ C_{J}\right)$. This assumes a $\mathrm{N}(0,100)$ prior on the difference, or roughly a $16 \%$ chance that model $i$ would be decisively better than model $j\left(\right.$ i.e. $\left.\left|B I C_{i}-B I C_{j}\right|>10\right)$. We then proceeded to choose the best-fitting model in a stepwise fashion: starting with a model with no annotations, we selected a model with more annotations in favor a model with fewer if the larger model was a considerably better fit-i.e. $\Delta_{i j}>2$. Model 1 was the best fit according to this process for overall and ER-positive breast cancer; Model 0 was the best fit for ERnegative breast cancer.

Differences between the PAINTOR and CCV outputs may be due to several factors. By considering functional enrichment and joint LD among all SNPs, PAINTOR may refine the set of likely causal variants; rather than imposing a hard threshold, PAINTOR allows for a gradient of evidence supporting causality; and the two sets of calculations are based on different summary statistics, CCV analyses used both iCOGS and OncoArray genotypes, while PAINTOR used only OncoArray data (Figure 1, Methods).

\section{Variant annotation}

Variants genome coordinates were converted to assembly GRCh38 with liftOver and uploaded to Variant Effect Predictor ${ }^{91}$ to determine their effect on genes, transcripts, and protein sequence. The commercial software Alamut ${ }^{\circledR}$ Batch v1.6 batch was also used to annotate coding and splicing variants. PolyPhen-2 ${ }^{92}$, SIFT ${ }^{93}$, MAPP ${ }^{94}$ were used to predict the consequence of missense coding variants. MaxEntScan ${ }^{95}$, Splice-Site Finder, and Human Splicing Finder ${ }^{96}$ were used to predict splicing effects. 


\section{INQUISIT analysis}

\section{Logic underlying INQUISIT predictions}

Briefly, genes were considered to potential targets of risk variants when variants affect: (1) distal regulation, (2) proximal genomic information, including chromatin interactions from capture $\mathrm{Hi}-\mathrm{C}$ experiments performed in a panel of six breast cell lines ${ }^{97}$, chromatin interaction analysis by pairedend tag sequencing (ChIA-PET; $\left.{ }^{8}\right)$ and genome-wide chromosome conformation capture from HMECS (Hi-C, (Rao et al., 2014)). We used computational enhancer-promoter correlations (PreSTIGE ${ }^{99}$, IM-PET (He et al., 2014), FANTOM5 ${ }^{100}$ and super-enhancers ${ }^{28}$ ), results for breast tissue-specific expression variants (eVariants) from multiple independent studies (TCGA, METABRIC, NHS, Methods), allelespecific imbalance in gene expression ${ }^{101}$, transcription factor and histone modification chromatin immunoprecipitation followed by sequencing (ChIP-Seq) from the ENCODE and Roadmap Epigenomics Projects together with the genomic features found to be significantly enriched as described above, gene expression RNA-seq from several breast cancer lines and normal samples and topologically associated domain (TAD) boundaries from T47D cells (ENCODE, ${ }^{102}$, Methods and Key Resources Table )). To assess the impact of intragenic variants, we evaluated their potential to alter splicing using Alamut@ Batch to identify new and cryptic donors and acceptors, and several tools to predict effects of coding sequence changes (Methods). The output from each tool was converted to a binary measure to indicate deleterious or tolerated predictions.

\section{Scoring hierarchy}

Each target gene prediction category (distal, promoter or coding) was scored according to different criteria. Genes predicted to be distally-regulated targets of CCVs were awarded points based on physical 
links (eg $\mathrm{CHi}-\mathrm{C}$ ), computational prediction methods, allele-specific expression, or eVariant associations. All CCV and HPPVs were considered as potentially involved in distal regulation. Intersection of a putative distal enhancer with genomic features found to be significantly enriched (see 'Genomic features enrichment' for details) were further upweighted. CCVs and HPPVs in gene proximal regulatory regions were intersected with histone ChIP-Seq peaks characteristic of promoters and assigned to the overlapping transcription start sites (defined as $-1.0 \mathrm{~kb}-+0.1 \mathrm{~kb}$ ). Further points were awarded to such genes if there was evidence for eVariant association or allele-specific expression, while a lack of expression resulted in down-weighting as potential targets. Potential coding changes including missense, nonsense and predicted splicing alterations resulted in addition of one point to the encoded gene for each type of change, while lack of expression reduced the score. We added an additional point for predicted target genes that were also breast cancer drivers. For each category, scores ranged from 0-7 (distal); 0-3 (promoter) or 0-2 (coding). We converted these scores into 'confidence levels': Level 1 (highest confidence) when distal score $>4$, promoter score $>=3$ or coding score $>1$; Level 2 when distal score $<=4$ and $>=1$, promoter score $=1$ or $=2$, coding score $=1$; and Level 3 when distal score $<1$ and $>0$, promoter score $<1$ and $>0$, and coding $<1$ and $>0$. For genes with multiple scores (for example, predicted as targets from multiple independent risk signals or predicted to be impacted in several categories), we recorded the highest score. Driver and transcription factor gene enrichment analysis was carried out using INQUISIT scores prior to adding a point for driver gene status. Modifications to the pipeline since original publication ${ }^{2}$ include:

- TAD boundary definitions from ENCODE T47D Hi-C analysis. Previously, we used regions from Rao, Cell 2013;

- eQTL: Addition of NHS normal and tumor samples 
- allele-specific imbalance using TCGA and GTEx RNA-seq data ${ }^{101}$

- Capture Hi-C data from six breast cell lines (Beesley et al.)

- Additional biofeatures derived from global enrichment in this study.

\section{Multi-signal targets}

To test if more genes were targeted by multiple signals than expected by chance, we modelled the number of signals per gene by negative binomial regression ( $R$ function glm.nb, package MASS) and Poisson regression ( $\mathrm{R}$ function $\mathrm{g} / \mathrm{m}$, package stats) with ChIA-PET interactions as a covariate and adjusted by fine mapping region. Likelihood ratio tests were used to compare goodness of fit. Rootograms were created using the $\mathrm{R}$ function rootogram (package vcd).

\section{Pathway analysis}

The pathway gene set database, dated 1 September 2018 was used 103 (http://download.baderlab.org/EM Genesets/current release/Human/symbol/). This database contains pathways from Reactome ${ }^{104}$, NCl Pathway Interaction Database ${ }^{105}$, GO (Gene Ontology) ${ }^{106}$, HumanCyc ${ }^{107}$, MSigdb $^{108}$, NetPath ${ }^{109}$, and Panther ${ }^{110}$. All duplicated pathways, defined in two or more databases, were included. To provide more biologically meaningful results, only pathways that contained $\leq 200$ genes were used.

We interrogated the pathway annotation sets with the list of high-confidence (Level 1) INQUISIT gene list. The significance of over-representation of the INQUISIT genes within each pathway was assessed with a hypergeometric test, ${ }^{111}$, using the R function phyper as follows: 


$$
P(x \mid n, m, N)=1-\sum_{i=0}^{x-1} \frac{\left(\begin{array}{c}
m \\
i
\end{array}\right)\left(\begin{array}{c}
N-m \\
n-i
\end{array}\right)}{\left(\begin{array}{c}
N \\
n
\end{array}\right)}
$$

where $\mathrm{x}$ is the number of Level 1 genes that overlap with any of the genes in the pathway, $\mathrm{n}$ is the number of genes in the pathway, $m$ is the number of Level1 genes that overlap with any of the genes

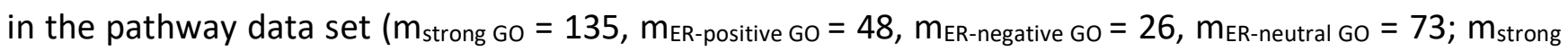

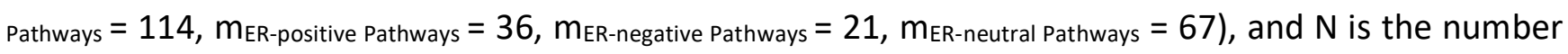
of genes in the pathway data set $\left(N_{G e n e s ~}^{G O}=14,252, N_{\text {Genes Pathways }}=10,915\right)$. We only included pathways that overlapped with at least two Level 1 genes. We used the Benjamini-Hochberg false discovery rate (FDR) ${ }^{62}$ at $5 \%$ level.

\section{ACKNOWLEDGMENTS}

We thank all the individuals who took part in these studies and all the researchers, clinicians, technicians and administrative staff who have enabled this work to be carried out. This work was supported by the European Union's Horizon 2020 research and innovation programme under the Marie Sklodowska-Curie grant agreement No 656144. Genotyping of the OncoArray was principally funded from three sources: the PERSPECTIVE project, funded by the Government of Canada through Genome Canada and the Canadian Institutes of Health Research, the 'Ministère de l'Économie, de la Science et de l'Innovation du Québec' through Genome Québec, and the Quebec Breast Cancer Foundation; the $\mathrm{NCl}$ Genetic Associations and Mechanisms in Oncology (GAME-ON) initiative and Discovery, Biology and Risk of Inherited Variants in Breast Cancer (DRIVE) project (NIH Grants U19 CA148065 and X01HG007492); and Cancer Research UK (C1287/A10118 and C1287/A16563). BCAC is funded by 
Cancer Research UK (C1287/A16563), by the European Community's Seventh Framework Programme under grant agreement 223175 (HEALTH-F2-2009-223175) (COGS) and by the European Union's Horizon 2020 Research and Innovation Programme under grant agreements 633784 (B-CAST) and 634935 (BRIDGES). Genotyping of the iCOGS array was funded by the European Union (HEALTH-F22009-223175), Cancer Research UK (C1287/A10710), the Canadian Institutes of Health Research for the 'ClHR Team in Familial Risks of Breast Cancer' program, and the Ministry of Economic Development, Innovation and Export Trade of Quebec, grant PSR-SIIRI-701. Combining of the GWAS data waS supported in part by The National Institute of Health (NIH) Cancer Post-Cancer GWAS initiative grant U19 CA 148065 (DRIVE, part of the GAME-ON initiative). For a full description of funding and acknowledgments, see Supplementary Note.

\section{AUTHOR CONTRIBUTIONS}

Conceptualization: L.Fa., H.As., J.Be., D.R.B., J.Al., S.Ka., K.A.P., K.Mi., P.So., A.Le., M.Gh., P.D.P.P., J.C.C., M.G.C., M.K.S., R.L.M., V.N.K., J.D.E., S.L.E., A.C.A., G.C.T., J.Si., D.F.E., P.K., A.M.D. Methodology: L.Fa.,, H.As., J.Be., D.R.B., J.Al., J.D.E., S.L.E., A.C.A., G.C.T., J.Si., D.F.E., P.K., A.M.D. Software: J.Be., J.P.T., M.L. Formal analysis: L.Fa., H.As., J.Be., D.R.B., J.Al., S.Ka., C.Tu., M.Mor., X.J. Resources: S.A., K.A., M.R.A., I.L.A., H.A.C., N.N.A., A.A., V.A., K.J.A., B.K.A., B.A., P.L.A., J.Az., J.Ba., R.B.B., D.B., A.B.F., J.Ben., M.B., K.B., A.M.B., C.B., W.B., N.V.B., S.E.B., B.Bo., A.B., H.Bra., H.Bre., I.B., I.W.B., A.B.W., T.B., B.Bu., S.S.B., Q.C., T.C., M.A.C., N.J.C., I.C., F.C., J.S.C., B.D.C., J.E.C., J.C., H.C., W.K.C., K.B.M., C.L.C., J.M.C., S.C., F.J.C., A.C., S.S.C., C.C., K.C., M.B.D., M.D.H., P.D., O.D., Y.C.D., G.S.D., S.M.D., T.D., I.D.S., A.D., S.D., M.Dum., M.Dur., L.D., M.Dw., D.M.E., C.E., M.E., D.G.E., P.A.F., U.F., O.F., G.F., H.F., L.Fo., W.D.F., E.F., L.Fr., D.F., M.Ga., M.G.D., G.Ga., P.A.G., S.M.G., J.Ga., J.A.G., M.M.G., V.G., G.G.G., G.Gl., A.K.G., M.S.G., D.E.G., 
A.G.N., M.H.G., M.Gr., J.Gr., A.G., P.G., E.H., C.A.H., N.H., P.Ha., U.H., P.A.H., J.M.H., M.H., W.H., C.S.H., B.A.M., J.H., P.Hi., F.B.L., A.H., M.J.H., J.L.H., A.Ho., G.H., P.J.H., E.N.I., C.I., M.I., A.Jag., M.J., A.Jak., P.J., R.J., R.C.J., E.M.J., N.J., M.E.J., A.Juk., A.Jun., R.Ka., D.K., B.Pes., R.Ke., M.J.K., E.K., J.I.K., J.K., C.M.K., Y.K., I.K., V.K., S.Ko., K.K.S., T.K., A.K., K.K., Y.L., D.L., E.L., G.L., J.Le., F.L., A.Li., W.L., J.Lo., A.Lo., J.T.L., J.Lu., R.J.M., T.M., E.M., A.Ma., M.Ma., S.Man., S.Mag., M.E.M., K.Ma., D.M., R.M., L.M., C.M., N.Me., A.Me., P.M., A.Mi., N.Mi., M.Mo., F.M., A.M.M., V.M.M., T.A., S.A.N., R.N., K.L.N., N.Z.N., H.N., P.N., F.C.N., L.N.Z., A.N., K.O., E.O., O.I.O., H.O., N.O., A.O., V.S.P., J.Pa., S.K.P., T.W.P.S., M.T.P., J.Pau., I.S.P., B.Pei., B.Y.K., P.P., J.Pe., D.P.K., K.Pr., R.P., N.P., D.P., M.A.P., K.Py., P.R., S.J.R., J.R., R.R.M., G.R., H.A.R., M.R., A.R., C.M.R., E.S., E.S.H., D.P.S., M.Sa., C.Sa., E.J.S., M.T.S., D.F.S., R.K.S., A.S., M.J.S., B.S., P.Sc., C.Sc., R.J.S., L.S., C.M.D., M.Sh., P.Sh., C.Y.S., X.S., C.F.S., T.P.S., S.S., M.C.S., J.J.S., A.B.S., J.St., D.S.L., C.Su., A.J.S., R.M.T., Y.Y.T., W.J.T., J.A.T., M.R.T., M.Te., S.H., M.B.T., A.T., M.Th., D.L.T., M.G.T., M.Ti., A.E.T., R.A.E., I.T., D.T., G.T.M., M.A.T., N.T., M.Tz., H.U.U., C.M.V., C.J.A., L.E.K., E.J.R., A.Ve., A.Vi., J.V., M.J.V., Q.W., B.W., C.R.W., J.N.W., C.W., H.W., R.W., A.W., A.H.W., D.Y., Y.Z., W.Z. Data management and curation: K.Mi., J.D., M.K.B., Q.W., R.Ke., J.C.C. and M.K.S. Writing original draft: L.Fa., H.As., J.Be., G.C.T., D.F.E., P.K., A.M.D. Writing review and editing: D.R.B., J.Al., P.So., A.Le., V.N.K., J.D.E., S.L.E., A.C.A., J.Si. Visualization: L.Fa., H.As., J.Be., C.Tu. Supervision: A.C.A., G.C.T., J.Si., D.F.E., P.K., A.M.D. Funding acquisition: L.Fa., P.D.P.P., J.C.C., M.G.C., M.K.S., R.L.M., V.N.K., J.D.E., S.L.E., A.C.A., G.C.T., J.Si., D.F.E., P.K., A.M.D. All authors read and approved the final version of the manuscript.

\section{COMPETING INTERESTS STATEMENT}

The authors declare no competing interests. 


\section{MATERIALS \& CORRESPONDENCE}

Further information and requests for resources should be directed to and will be fulfilled by Manjeet Bolla (bcac@medschl.cam.ac.uk)

\section{REFERENCES}

1. Milne, R.L. et al. Identification of ten variants associated with risk of estrogen-receptor-negative breast cancer. Nat Genet 49, 1767-1778 (2017).

2. Michailidou, K. et al. Association analysis identifies 65 new breast cancer risk loci. Nature 551, 9294 (2017).

3. Ghoussaini, M. et al. Evidence that breast cancer risk at the $2 q 35$ locus is mediated through IGFBP5 regulation. Nat Commun 4, 4999 (2014).

4. Wyszynski, A. et al. An intergenic risk locus containing an enhancer deletion in 2 q35 modulates breast cancer risk by deregulating IGFBP5 expression. Hum Mol Genet 25, 3863-3876 (2016).

5. Guo, X. et al. Fine-scale mapping of the $4 q 24$ locus identifies two independent loci associated with breast cancer risk. Cancer Epidemiol Biomarkers Prev 24, 1680-91 (2015).

6. Glubb, D.M. et al. Fine-scale mapping of the $5 q 11.2$ breast cancer locus reveals at least three independent risk variants regulating MAP3K1. Am J Hum Genet 96, 5-20 (2015).

7. Dunning, A.M. et al. Breast cancer risk variants at $6 q 25$ display different phenotype associations and regulate ESR1, RMND1 and CCDC170. Nat Genet 48, 374-86 (2016).

8. Shi, J. et al. Fine-scale mapping of $8 q 24$ locus identifies multiple independent risk variants for breast cancer. Int J Cancer 139, 1303-1317 (2016). 
9. Orr, N. et al. Fine-mapping identifies two additional breast cancer susceptibility loci at 9q31.2. Hum Mol Genet 24, 2966-84 (2015).

10. Darabi, H. et al. Polymorphisms in a Putative Enhancer at the $10 q 21.2$ Breast Cancer Risk Locus Regulate NRBF2 Expression. Am J Hum Genet 97, 22-34 (2015).

11. Darabi, H. et al. Fine scale mapping of the $17 q 22$ breast cancer locus using dense SNPs, genotyped within the Collaborative Oncological Gene-Environment Study (COGs). Sci Rep 6, 32512 (2016).

12. Meyer, K.B. et al. Fine-scale mapping of the FGFR2 breast cancer risk locus: putative functional variants differentially bind FOXA1 and E2F1. Am J Hum Genet 93, 1046-60 (2013).

13. Betts, J.A. et al. Long noncoding RNAs CUPID1 and CUPID2 mediate breast cancer risk at $11 q 13$ by modulating the response to dna damage. Am J Hum Genet 101, 255-266 (2017).

14. French, J.D. et al. Functional variants at the $11 q 13$ risk locus for breast cancer regulate cyclin D1 expression through long-range enhancers. Am J Hum Genet 92, 489-503 (2013).

15. Ghoussaini, M. et al. Evidence that the $5 p 12$ variant rs10941679 Confers susceptibility to estrogen-receptor-positive breast cancer through FGF10 and MRPS30 regulation. Am J Hum Genet 99, 903-911 (2016).

16. Horne, H.N. et al. Fine-mapping of the 1p11.2 Breast cancer susceptibility locus. PLoS One 11, e0160316 (2016).

17. Zeng, C. et al. Identification of independent association signals and putative functional variants for breast cancer risk through fine-scale mapping of the 12p11 locus. Breast Cancer Res 18, 64 (2016).

18. Lin, W.Y. et al. Identification and characterization of novel associations in the CASP8/ALS2CR12 region on chromosome 2 with breast cancer risk. Hum Mol Genet 24, 285-98 (2015). 
19. Bojesen, S.E. et al. Multiple independent variants at the TERT locus are associated with telomere length and risks of breast and ovarian cancer. Nat Genet 45, 371-84, 384e1-2 (2013).

20. Lawrenson, K. et al. Functional mechanisms underlying pleiotropic risk alleles at the $19 p 13.1$ breast-ovarian cancer susceptibility locus. Nat Commun 7, 12675 (2016).

21. Amos, C.I. et al. The OncoArray Consortium: A Network for Understanding the Genetic Architecture of Common Cancers. Cancer Epidemiol Biomarkers Prev 26, 126-135 (2017).

22. Michailidou, K. et al. Large-scale genotyping identifies 41 new loci associated with breast cancer risk. Nat Genet 45, 353-61, 361e1-2 (2013).

23. Michailidou, K. et al. Genome-wide association analysis of more than 120,000 individuals identifies 15 new susceptibility loci for breast cancer. Nature Genetics 47, 373-U127 (2015).

24. Udler, M.S., Tyrer, J. \& Easton, D.F. Evaluating the power to discriminate between highly correlated SNPs in genetic association studies. Genet Epidemiol 34, 463-8 (2010).

25. Mavaddat, N., Antoniou, A.C., Easton, D.F. \& Garcia-Closas, M. Genetic susceptibility to breast cancer. Mol Oncol 4, 174-91 (2010).

26. Lakhani, S.R. et al. Prediction of BRCA1 status in patients with breast cancer using estrogen receptor and basal phenotype. Clin Cancer Res 11, 5175-80 (2005).

27. Taberlay, P.C., Statham, A.L., Kelly, T.K., Clark, S.J. \& Jones, P.A. Reconfiguration of nucleosomedepleted regions at distal regulatory elements accompanies DNA methylation of enhancers and insulators in cancer. Genome Res 24, 1421-32 (2014).

28. Hnisz, D. et al. Super-enhancers in the control of cell identity and disease. Cell 155, 934-47 (2013).

29. Farh, K.K. et al. Genetic and epigenetic fine mapping of causal autoimmune disease variants. Nature 518, 337-43 (2015). 
30. Cowper-Sal lari, R. et al. Breast cancer risk-associated SNPs modulate the affinity of chromatin for FOXA1 and alter gene expression. Nat Genet 44, 1191-8 (2012).

31. Kichaev, G. et al. Integrating functional data to prioritize causal variants in statistical fine-mapping studies. PLoS Genet 10, e1004722 (2014).

32. Quiroz-Zarate, A. et al. Expression Quantitative Trait loci (QTL) in tumor adjacent normal breast tissue and breast tumor tissue. PLoS One 12, e0170181 (2017).

33. Cancer Genome Atlas Research, N. et al. The Cancer Genome Atlas Pan-Cancer analysis project. Nat Genet 45, 1113-20 (2013).

34. Curtis, C. et al. The genomic and transcriptomic architecture of 2,000 breast tumours reveals novel subgroups. Nature 486, 346-52 (2012).

35. Ciriello, G. et al. Comprehensive Molecular Portraits of Invasive Lobular Breast Cancer. Cell 163, 506-19 (2015).

36. Nik-Zainal, S. et al. Landscape of somatic mutations in 560 breast cancer whole-genome sequences. Nature 534, 47-54 (2016).

37. Pereira, B. et al. The somatic mutation profiles of 2,433 breast cancers refines their genomic and transcriptomic landscapes. Nat Commun 7, 11479 (2016).

38. Cancer Genome Atlas, N. Comprehensive molecular portraits of human breast tumours. Nature 490, $61-70$ (2012).

39. Bailey, M.H. et al. Comprehensive Characterization of Cancer Driver Genes and Mutations. Cell 173, 371-385 e18 (2018).

40. Lambert, S.A. et al. The Human Transcription Factors. Cell 172, 650-665 (2018). 
41. Artero-Castro, A. et al. Disruption of the ribosomal P complex leads to stress-induced autophagy. Autophagy 11, $1499-519$ (2015).

42. Wang, X.Y. et al. Musashi1 modulates mammary progenitor cell expansion through proliferinmediated activation of the Wnt and Notch pathways. Mol Cell Biol 28, 3589-99 (2008).

43. Vijayan, D., Young, A., Teng, M.W.L. \& Smyth, M.J. Targeting immunosuppressive adenosine in cancer. Nat Rev Cancer 17, 709-724 (2017).

44. Takebe, N. et al. Targeting Notch, Hedgehog, and Wnt pathways in cancer stem cells: clinical update. Nat Rev Clin Oncol 12, 445-64 (2015).

45. Thorpe, L.M., Yuzugullu, H. \& Zhao, J.J. PI3K in cancer: divergent roles of isoforms, modes of activation and therapeutic targeting. Nat Rev Cancer 15, 7-24 (2015).

46. Nusse, R. \& Clevers, H. Wnt/beta-Catenin Signaling, Disease, and Emerging Therapeutic Modalities. Cell 169, 985-999 (2017).

47. Massague, J. TGFbeta signalling in context. Nat Rev Mol Cell Biol 13, 616-30 (2012).

48. Meeks, H.D. et al. BRCA2 polymorphic stop codon K3326X and the Risk of breast, prostate, and ovarian cancers. J Natl Cancer Inst 108(2016).

49. CHEK2 Breast Cancer Case-Control Consortium. CHEK2*1100delC and susceptibility to breast cancer: a collaborative analysis involving 10,860 breast cancer cases and 9,065 controls from 10 studies. Am J Hum Genet 74, 1175-82 (2004).

50. Schmidt, M.K. et al. Age- and Tumor Subtype-Specific Breast Cancer Risk Estimates for CHEK2*1100delC Carriers. J Clin Oncol 34, 2750-60 (2016).

51. Kilpivaara, O. et al. CHEK2 variant I157T may be associated with increased breast cancer risk. Int J Cancer 111, 543-7 (2004). 
52. Muranen, T.A. et al. Patient survival and tumor characteristics associated with CHEK2:p.I157T findings from the Breast Cancer Association Consortium. Breast Cancer Res 18, 98 (2016).

53. Killedar, A. et al. A Common Cancer Risk-Associated Allele in the hTERT Locus Encodes a Dominant Negative Inhibitor of Telomerase. PLoS Genet 11, e1005286 (2015).

54. De Blasio, A. et al. Unusual roles of caspase-8 in triple-negative breast cancer cell line MDA-MB231. Int J Oncol 48, 2339-48 (2016).

55. Haupt, S. et al. Targeting Mdmx to treat breast cancers with wild-type p53. Cell Death Dis 6, e1821 (2015).

56. Pandya, P.H., Murray, M.E., Pollok, K.E. \& Renbarger, J.L. The Immune System in Cancer Pathogenesis: Potential Therapeutic Approaches. J Immunol Res 2016, 4273943 (2016).

57. Gionet, N., Jansson, D., Mader, S. \& Pratt, M.A. NF-kappaB and estrogen receptor alpha interactions: Differential function in estrogen receptor-negative and -positive hormoneindependent breast cancer cells. J Cell Biochem 107, 448-59 (2009).

58. Fleischer, T. et al. DNA methylation at enhancers identifies distinct breast cancer lineages. Nat Commun 8, 1379 (2017).

59. Couch, F.J. et al. Genome-wide association study in BRCA1 mutation carriers identifies novel loci associated with breast and ovarian cancer risk. PLoS Genet 9, e1003212 (2013).

60. Gaudet, M.M. et al. Identification of a BRCA2-specific modifier locus at $6 \mathrm{p} 24$ related to breast cancer risk. PLoS Genet 9, e1003173 (2013).

61. Marchini, J., Howie, B., Myers, S., McVean, G. \& Donnelly, P. A new multipoint method for genome-wide association studies by imputation of genotypes. Nat Genet 39, 906-13 (2007). 
62. Benjamini, Y. \& Hochberg, Y. Controlling the False Discovery Rate - a Practical and Powerful Approach to Multiple Testing. Journal of the Royal Statistical Society Series B-Methodological 57, 289-300 (1995).

63. Liu, J.Z. et al. Meta-analysis and imputation refines the association of $15 q 25$ with smoking quantity. Nat Genet 42, 436-40 (2010).

64. Antoniou, A.C. et al. RAD51 135G-->C modifies breast cancer risk among BRCA2 mutation carriers: results from a combined analysis of 19 studies. Am J Hum Genet 81, 1186-200 (2007).

65. Barnes, D.R. et al. Evaluation of association methods for analysing modifiers of disease risk in carriers of high-risk mutations. Genet Epidemiol 36, 274-91 (2012).

66. Willer, C.J., Li, Y. \& Abecasis, G.R. METAL: fast and efficient meta-analysis of genomewide association scans. Bioinformatics 26, 2190-1 (2010).

67. Hunter, D.J. et al. A genome-wide association study identifies alleles in FGFR2 associated with risk of sporadic postmenopausal breast cancer. Nat Genet 39, 870-4 (2007).

68. Baran, Y. et al. Fast and accurate inference of local ancestry in Latino populations. Bioinformatics 28, 1359-67 (2012).

69. Howie, B., Fuchsberger, C., Stephens, M., Marchini, J. \& Abecasis, G.R. Fast and accurate genotype imputation in genome-wide association studies through pre-phasing. Nat Genet 44, 955-9 (2012).

70. Genomes Project, C. et al. An integrated map of genetic variation from 1,092 human genomes. Nature 491, 56-65 (2012).

71. Li, B. \& Dewey, C.N. RSEM: accurate transcript quantification from RNA-Seq data with or without a reference genome. BMC Bioinformatics 12, 323 (2011). 
72. Mermel, C.H. et al. GISTIC2.0 facilitates sensitive and confident localization of the targets of focal somatic copy-number alteration in human cancers. Genome Biol 12, R41 (2011).

73. Li, Q. et al. Integrative eQTL-based analyses reveal the biology of breast cancer risk loci. Cell 152, 633-41 (2013).

74. Shabalin, A.A. Matrix eQTL: ultra fast eQTL analysis via large matrix operations. Bioinformatics 28, 1353-8 (2012).

75. ENCODE Project Consortium. An integrated encyclopedia of DNA elements in the human genome. Nature 489, 57-74 (2012).

76. Sloan, C.A. et al. ENCODE data at the ENCODE portal. Nucleic Acids Res 44, D726-32 (2016).

77. Roadmap Epigenomics, C. et al. Integrative analysis of 111 reference human epigenomes. Nature 518, 317-30 (2015).

78. Stunnenberg, H.G., International Human Epigenome, C. \& Hirst, M. The International Human Epigenome Consortium: A Blueprint for Scientific Collaboration and Discovery. Cell 167, 1897 (2016).

79. Pellacani, D. et al. Analysis of Normal Human Mammary Epigenomes Reveals Cell-Specific Active Enhancer States and Associated Transcription Factor Networks. Cell Rep 17, 2060-2074 (2016).

80. Cheneby, J., Gheorghe, M., Artufel, M., Mathelier, A. \& Ballester, B. ReMap 2018: an updated atlas of regulatory regions from an integrative analysis of DNA-binding ChIP-seq experiments. Nucleic Acids Res 46, D267-D275 (2018).

81. Pruitt, K.D. et al. RefSeq: an update on mammalian reference sequences. Nucleic Acids Res 42, D756-63 (2014). 
82. Harrow, J. et al. GENCODE: the reference human genome annotation for The ENCODE Project. Genome Res 22, 1760-74 (2012).

83. Wang, J. et al. Sequence features and chromatin structure around the genomic regions bound by 119 human transcription factors. Genome Res 22, 1798-812 (2012).

84. Mathelier, A. et al. JASPAR 2016: a major expansion and update of the open-access database of transcription factor binding profiles. Nucleic Acids Res 44, D110-5 (2016).

85. Tan, G. \& Lenhard, B. TFBSTools: an R/bioconductor package for transcription factor binding site analysis. Bioinformatics 32, 1555-6 (2016).

86. Grant, C.E., Bailey, T.L. \& Noble, W.S. FIMO: scanning for occurrences of a given motif. Bioinformatics 27, 1017-8 (2011).

87. Grassi, E., Zapparoli, E., Molineris, I. \& Provero, P. Total Binding Affinity Profiles of Regulatory Regions Predict Transcription Factor Binding and Gene Expression in Human Cells. PLoS One 10, e0143627 (2015).

88. Quinlan, A.R. \& Hall, I.M. BEDTools: a flexible suite of utilities for comparing genomic features. Bioinformatics 26, 841-2 (2010).

89. McLeay, R.C. \& Bailey, T.L. Motif Enrichment Analysis: a unified framework and an evaluation on ChIP data. BMC Bioinformatics 11, 165 (2010).

90. Kichaev, G. et al. Improved methods for multi-trait fine mapping of pleiotropic risk loci. Bioinformatics 33, 248-255 (2017).

91. McLaren, W. et al. The Ensembl Variant Effect Predictor. Genome Biol 17, 122 (2016).

92. Adzhubei, I.A. et al. A method and server for predicting damaging missense mutations. Nat Methods 7, 248-9 (2010). 
93. Kumar, P., Henikoff, S. \& Ng, P.C. Predicting the effects of coding non-synonymous variants on protein function using the SIFT algorithm. Nat Protoc 4, 1073-81 (2009).

94. Stone, E.A. \& Sidow, A. Physicochemical constraint violation by missense substitutions mediates impairment of protein function and disease severity. Genome Res 15, 978-86 (2005).

95. Yeo, G. \& Burge, C.B. Maximum entropy modeling of short sequence motifs with applications to RNA splicing signals. J Comput Biol 11, 377-94 (2004).

96. Desmet, F.O. et al. Human Splicing Finder: an online bioinformatics tool to predict splicing signals. Nucleic Acids Res 37, e67 (2009).

97. Beesley, J. et al. Chromatin interactome mapping at 139 independent breast cancer risk signals. Submitted.

98. Fullwood, M.J. et al. An oestrogen-receptor-alpha-bound human chromatin interactome. Nature 462, 58-64 (2009).

99. Corradin, O. et al. Combinatorial effects of multiple enhancer variants in linkage disequilibrium dictate levels of gene expression to confer susceptibility to common traits. Genome Res 24, 1-13 (2014).

100. Andersson, R. et al. An atlas of active enhancers across human cell types and tissues. Nature 507, 455-461 (2014).

101. Moradi Marjaneh, M. et al. High-throughput allelic expression imbalance analyses identify 14 candidate breast cancer risk genes. Submitted.

102. Dixon, J.R. et al. Integrative detection and analysis of structural variation in cancer genomes. Nat Genet 50, 1388-1398 (2018). 
103. Merico, D., Isserlin, R. \& Bader, G.D. Visualizing gene-set enrichment results using the Cytoscape plug-in enrichment map. Methods Mol Biol 781, 257-77 (2011).

104. Vastrik, I. et al. Reactome: a knowledge base of biologic pathways and processes. Genome Biol 8, R39 (2007).

105. Schaefer, C.F. et al. PID: the Pathway Interaction Database. Nucleic Acids Res 37, D674-9 (2009).

106. Ashburner, M. et al. Gene ontology: tool for the unification of biology. The Gene Ontology Consortium. Nat Genet 25, 25-9 (2000).

107. Romero, P. et al. Computational prediction of human metabolic pathways from the complete human genome. Genome Biol 6, R2 (2005).

108. Subramanian, A. et al. Gene set enrichment analysis: a knowledge-based approach for interpreting genome-wide expression profiles. Proc Natl Acad Sci U S A 102, 15545-50 (2005).

109. Kandasamy, K. et al. NetPath: a public resource of curated signal transduction pathways. Genome Biol 11, R3 (2010).

110. Thomas, P.D. et al. PANTHER: a library of protein families and subfamilies indexed by function. Genome Res 13, 2129-41 (2003).

111. Herwig, R., Hardt, C., Lienhard, M. \& Kamburov, A. Analyzing and interpreting genome data at the network level with ConsensusPathDB. Nat Protoc 11, 1889-907 (2016). 
Table 1. Signals with single CCVs and variants with PP > 80\%

\begin{tabular}{|c|c|c|c|c|c|c|c|c|c|c|c|c|c|c|c|}
\hline \multirow[b]{2}{*}{$\begin{array}{l}\text { Fine-mapping } \\
\text { region }^{a}\end{array}$} & \multirow[b]{2}{*}{ Variant ${ }^{b}$} & \multirow[b]{2}{*}{ Ref/Alt ${ }^{c}$} & \multirow[b]{2}{*}{ EAF $^{d}$} & \multirow[b]{2}{*}{ Ppe } & \multirow[b]{2}{*}{ Modelf $^{f}$} & \multirow[b]{2}{*}{ Signalg } & \multirow[b]{2}{*}{$\begin{array}{l}\mathrm{N} \\
\mathrm{CCV}^{\mathrm{h}}\end{array}$} & \multicolumn{2}{|c|}{ ER-negative } & \multicolumn{2}{|c|}{ ER-positive } & \multirow[b]{2}{*}{ P-value ${ }^{i}$} & \multirow[b]{2}{*}{$\mathrm{FP}^{\mathrm{j}}$} & \multirow[b]{2}{*}{$\begin{array}{l}\text { Predicted } \\
\text { target gene }(s)^{k}\end{array}$} & \multirow[b]{2}{*}{ Confidence } \\
\hline & & & & & & & & $O^{i}$ & $(95 \% \mathrm{Cl})$ & $O^{i}$ & $(95 \% \mathrm{Cl})$ & & & & \\
\hline $\begin{array}{l}\text { chr1:120723447 } \\
-121780613\end{array}$ & rs11249433 & $A / G$ & 0.42 & 0.57 & ERALL & Signal 1 & 1 & 1.02 & $(0.99-1.04)$ & 1.13 & $(1.11-1.15)$ & $8.11 \times 10^{-60}$ & na & na & \\
\hline $\begin{array}{l}c h r 1: 200937832 \\
-201937832\end{array}$ & rs35383942 & $\mathrm{C} / \mathrm{T}$ & 0.06 & 0.96 & ERALL & Signal 1 & 2 & 1.10 & $(1.05-1.16)$ & 1.09 & $(1.06-1.13)$ & $1.14 \times 10^{-7}$ & D & TNNI1 & Level 1 \\
\hline $\begin{array}{l}\text { chr2:201681247 } \\
-202681247\end{array}$ & rs3769821 & $\mathrm{C} / \mathrm{T}$ & 0.66 & 0.40 & ERALL & Signal 1 & 1 & 0.94 & $(0.92-0.97)$ & 0.95 & $(0.93-0.96)$ & $1.46 \times 10^{-12}$ & D & $\begin{array}{ll}\text { ALS2CR11, } & \text { ALS2CR12, } \\
\text { MPP4, } & \text { NIF3L1, } \\
\text { TMEM237 } & \end{array}$ & $\begin{array}{l}\text { CASP8 } \\
\text { STRADB, Level } 2\end{array}$ \\
\hline $\begin{array}{l}\text { chr2:217405832 } \\
-218796508\end{array}$ & rs4442975 & $\mathrm{G} / \mathrm{T}$ & 0.48 & 0.84 & ERALL & Signal 1 & 1 & 0.94 & $(0.92-0.97)$ & 0.86 & $(0.85-0.87)$ & $2.50 \times 10^{-90}$ & D & IGFBP $5^{m}$ & Level 1 \\
\hline $\begin{array}{l}\text { chr4:105569013 } \\
-106856761\end{array}$ & esv3601665 & -/Alu & 0.07 & 0.95 & ERPOS & & & 1.01 & $(0.95-1.08)$ & 1.10 & $(1.06-1.14)$ & $3.27 \times 10^{-6}$ & D & ARHGEF38, AC004066.3 & Level 1 \\
\hline $\begin{array}{l}\text { chr5:779790 } \\
-1797488\end{array}$ & rs10069690 & $C / T$ & 0.27 & 0.58 & ERNEG & Signal 1 & 1 & 1.18 & $(1.15-1.21)$ & 1.03 & $(1.01-1.05)$ & $1.20 \times 10^{-34}$ & D & CLPTM1L, TERT ${ }^{m}$ & Level 1 \\
\hline \multirow[t]{2}{*}{$\begin{array}{l}\text { chr5:44013304 } \\
-45206498\end{array}$} & rs10941679 & $A / G$ & 0.26 & 0.00 & ERPOS & Signal 1 & 1 & 1.04 & $(1.02-1.07)$ & 1.17 & $(1.15-1.19)$ & $1.50 \times 10^{-77}$ & D & MRPS30 & Level 2 \\
\hline & rs5867671 & $A /-$ & 0.77 & 0.01 & ERPOS & Signal 2 & 1 & 0.91 & $(0.89-0.94)$ & 0.99 & $(0.97-1.01)$ & $2.25 \times 10^{-9}$ & na & na & \\
\hline $\begin{array}{l}\text { chr5:44013304 } \\
-45206498\end{array}$ & rs190443933 & $\mathrm{T} / \mathrm{C}$ & 0.01 & 0.00 & ERALL & Signal 4 & 1 & 1.30 & $(1.14-1.48)$ & 1.26 & $(1.16-1.37)$ & $2.32 \times 10^{-8}$ & na & na & \\
\hline \multirow[t]{2}{*}{$\begin{array}{l}\text { chr5:55531884 } \\
-56587883\end{array}$} & rs984113 & $\mathrm{G} / \mathrm{C}$ & 0.61 & 0.81 & ERPOS & Signal 2 & 1 & 0.96 & $(0.93-0.98)$ & 0.96 & $(0.94-0.97)$ & $3.51 \times 10^{-8}$ & D & $M A P 3 K 1^{m}$ & Level 1 \\
\hline & rs889310 & $\mathrm{C} / \mathrm{T}$ & 0.56 & 0.84 & ERPOS & (Signal 6) & 15 & 1.03 & $(1.00-1.05)$ & 1.05 & $(1.03-1.06)$ & $1.75 \times 10^{-7}$ & D & $M A P 3 K 1^{m}$ & Level 1 \\
\hline $\begin{array}{l}\text { chr6:15899557 } \\
-16899557\end{array}$ & rs3819405 & $\mathrm{C} / \mathrm{T}$ & 0.32 & 0.96 & ERALL & Signal 1 & 1 & 0.97 & $(0.95-1.00)$ & 0.95 & $(0.94-0.97)$ & $1.14 \times 10^{-7}$ & D & $\begin{array}{l}\text { ATXN1, RP1-151F17.1, } \\
\text { 151F17.2 }\end{array}$ & $\quad R P 1{ }^{-}$Level 2 \\
\hline \multirow[t]{3}{*}{$\begin{array}{l}\text { chr6:151418856 } \\
-152937016\end{array}$} & rs12173562 & $\mathrm{C} / \mathrm{T}$ & 0.08 & 0.10 & ERNEG & Signal 1 & 1 & 1.30 & $(1.25-1.36)$ & 1.14 & (1.11-1.18) & $3.98 \times 10^{-40}$ & D & $E S R 1^{m}$ & Level 1 \\
\hline & rs34133739 & $-/ C$ & 0.53 & 0.25 & ERALL & Signal 2 & 1 & 1.11 & (1.09-1.14) & 1.05 & (1.04-1.07) & $2.36 \times 10^{-22}$ & D & ESR $1^{m}$, SYNE1 & Level 2 \\
\hline & rs851984 & $\mathrm{G} / \mathrm{A}$ & 0.40 & 0.73 & ERALL & Signal 3 & 1 & 1.07 & (1.04-1.09) & 1.05 & $(1.04-1.07)$ & $3.69 \times 10^{-13}$ & D & $E S R 1^{m}$ & Level 1 \\
\hline $\begin{array}{l}\text { chr7:130167121 } \\
-131167121\end{array}$ & rs68056147 & $\mathrm{G} / \mathrm{A}$ & 0.30 & 0.84 & ERALL & & & 1.04 & $(1.01-1.07)$ & 1.05 & $(1.03-1.06)$ & $3.07 \times 10^{-7}$ & D & MKLN1 & Level 2 \\
\hline $\begin{array}{l}\text { chr8:127424659 } \\
-130041931\end{array}$ & rs35961416 & $-/ A$ & 0.41 & 0.68 & ERALL & Signal 3 & 1 & 0.97 & $(0.94-0.99)$ & 0.95 & $(0.93-0.96)$ & $9.97 \times 10^{-11}$ & D & CASC11, MYC & Level 2 \\
\hline $\begin{array}{l}\text { chr9:21247803 } \\
-22624477\end{array}$ & rs539723051 & AAAA/- & 0.33 & 0.43 & ERALL & Signal 1 & 1 & 1.08 & $(1.05-1.11)$ & 1.06 & $(1.04-1.08)$ & $1.81 \times 10^{-15}$ & na & na & \\
\hline
\end{tabular}




\begin{tabular}{|c|c|c|c|c|c|c|c|c|c|c|c|c|c|c|c|}
\hline $\begin{array}{l}\text { chr9:109803808 } \\
-111395353\end{array}$ & rs10816625 & $A / G$ & 0.07 & 0.95 & ERPOS & Signal 3 & 1 & 1.06 & $(1.01-1.11)$ & 1.13 & $(1.10-1.16)$ & $3.62 \times 10^{-15}$ & D & $K L F 4^{m}$ & Level 2 \\
\hline & rs13294895 & $C / T$ & 0.18 & 0.93 & ERPOS & Signal 4 & 1 & 1.01 & $(0.98-1.05)$ & 1.09 & $(1.07-1.11)$ & $4.00 \times 10^{-17}$ & D & $K L F 4^{m}$ & Level 1 \\
\hline $\begin{array}{l}\text { chr9:109803808 } \\
-111395353\end{array}$ & rs60037937 & $A A /-$ & 0.22 & 0.68 & ERPOS & Signal 2 & 1 & 1.02 & $(0.99-1.06)$ & 1.11 & $(1.09-1.13)$ & $3.17 \times 10^{-26}$ & D & $K L F 4^{m}, R A D 23 B$ & Level 2 \\
\hline $\begin{array}{l}\text { chr10:63758684 } \\
-65063702\end{array}$ & rs10995201 & $A / G$ & 0.15 & 0.31 & ERALL & Signal 1 & 1 & 0.91 & $(0.88-0.94)$ & 0.87 & $(0.85-0.89)$ & $1.40 \times 10^{-37}$ & na & na & \\
\hline $\begin{array}{l}\text { chr10:122593901 } \\
-123849324\end{array}$ & rs45631563 & $A / T$ & 0.04 & 0.93 & ERPOS & Signal 3 & 1 & 0.97 & $(0.92-1.03)$ & 0.76 & $(0.73-0.79)$ & $4.84 \times 10^{-44}$ & C & $F G F R 2^{m}$ & Level 1 \\
\hline \multirow[t]{3}{*}{$\begin{array}{l}\text { chr10:122593901 } \\
-123849324\end{array}$} & rs35054928 & $\mathrm{C} /-$ & 0.56 & 0.60 & ERALL & Signal 1 & 1 & 0.96 & $(0.94-0.98)$ & 0.74 & $(0.73-0.76)$ & $6.55 \times 10^{-342}$ & D & $F G F R 2^{m}$ & Level 1 \\
\hline & rs45631563 & $\mathrm{A} / \mathrm{T}$ & 0.04 & 0.93 & ERPOS & Signal 3 & 1 & 0.97 & $(0.92-1.03)$ & 0.76 & $(0.73-0.79)$ & $4.84 \times 10^{-44}$ & c & $F G F R 2^{m}$ & Level 1 \\
\hline & rs7899765 & $\mathrm{T} / \mathrm{C}$ & 0.06 & 0.02 & ERALL & Signal 5 & 1 & 1.01 & $(0.97-1.06)$ & 0.87 & $(0.84-0.90)$ & $2.21 \times 10^{-18}$ & D & $F G F R 2^{m}$ & Level 1 \\
\hline $\begin{array}{l}\text { chr11:68831418 } \\
-69879161\end{array}$ & rs78540526 & $\mathrm{C} / \mathrm{T}$ & 0.09 & 0.91 & ERPOS & Signal 1 & 1 & 1.01 & $(0.97-1.06)$ & 1.40 & $(1.36-1.44)$ & $2.77 \times 10^{-145}$ & D & CCND1 ${ }^{m}$, MYEOV & Level 1 \\
\hline $\begin{array}{l}\text { chr12:27639846 } \\
-29034415\end{array}$ & rs7297051 & $\mathrm{C} / \mathrm{T}$ & 0.23 & 0.23 & ERALL & Signal 1 & 1 & 0.87 & $(0.85-0.90)$ & 0.89 & $(0.88-0.91)$ & $3.12 \times 10^{-43}$ & D & $\begin{array}{l}\text { CCDC91 } 1^{m}, \\
967 K 21.1\end{array} \quad$ PTHLH & - Level 2 \\
\hline $\begin{array}{l}\text { chr12:115336522 } \\
-116336522\end{array}$ & rs35422 & $\mathrm{G} / \mathrm{A}$ & 0.57 & 0.58 & ERPOS & Signal 2 & 1 & 0.98 & $(0.96-1.01)$ & 1.05 & $(1.03-1.07)$ & $4.85 \times 10^{-10}$ & D & TBX3 & Level 1 \\
\hline $\begin{array}{l}\text { chr14:91341069 } \\
-92368623\end{array}$ & rs7153397 & $\mathrm{C} / \mathrm{T}$ & 0.70 & 0.81 & ERPOS & Signal 1 & 3 & 1.01 & $(0.99-1.04)$ & 1.06 & $(1.04-1.08)$ & $3.25 \times 10^{-11}$ & $\mathrm{D}, \mathrm{C}$ & $\begin{array}{l}C C D C 88 C, C T D-2547 L 24.4, \text { GPR68, } \\
R P 11-73 M 18.7, R P 11-895 M 11.3\end{array}$ & Level 2 \\
\hline $\begin{array}{l}\text { chr16:52038825 } \\
-53038825\end{array}$ & rs4784227 & $\mathrm{C} / \mathrm{T}$ & 0.27 & 0.95 & ERPOS & Signal 1 & 1 & 1.15 & $(1.12-1.18)$ & 1.26 & $(1.24-1.28)$ & $4.63 \times 10^{-160}$ & D & TOX3 ${ }^{m}$ & Level 1 \\
\hline $\begin{array}{l}\text { chr18:23832476 } \\
-25075396\end{array}$ & rs180952292 & $\mathrm{T} / \mathrm{C}$ & 0.01 & 0.01 & ERNEG & Signal 4 & 1 & 1.24 & $(1.12-1.37)$ & 0.98 & $(0.92-1.05)$ & $2.07 \times 10^{-5}$ & na & na & \\
\hline $\begin{array}{l}\text { chr18:41899590 } \\
-42899590\end{array}$ & rs9952980 & $\mathrm{T} / \mathrm{C}$ & 0.34 & 0.95 & ERALL & Signal 2 & 3 & 0.97 & (0.94-0.99) & 0.95 & $(0.93-0.96)$ & $7.43 \times 10^{-12}$ & D & SETBP 1 & Level 2 \\
\hline $\begin{array}{l}\text { chr20:5448227 } \\
-6448227\end{array}$ & rs16991615 & $\mathrm{G} / \mathrm{A}$ & 0.07 & 0.97 & ERALL & Signal 1 & 1 & 1.09 & (1.04-1.15) & 1.07 & $(1.04-1.11)$ & $7.89 \times 10^{-7}$ & D, C & GPCPD1, MCM8 & Level 2 \\
\hline $\begin{array}{l}\text { chr22:45783297 } \\
-46783297\end{array}$ & rs184070480 & $\mathrm{C} / \mathrm{T}$ & 0.01 & 0.00 & ERALL & Signal 2 & 1 & 1.40 & $(1.20-1.64)$ & 1.01 & $(0.91-1.12)$ & $5.02 \times 10^{-5}$ & $\mathrm{D}$ & ATXN10, WNT7B & Level 2 \\
\hline
\end{tabular}

\section{${ }^{a}$ GRCh37/hg19, bp}

\section{${ }^{\mathrm{b}}$ Current reference ID}

${ }^{\mathrm{C}}$ Reference (Ref) versus Alternative (Alt) Allele 
${ }^{d}$ Effect allele (Alt allele) frequency in OncoArray

e PP: Posterior probability. Largest posterior probability in all evaluated models

${ }^{f}$ Model where the variant reaches the largest posterior probability

g Signal where the variant is included. Between brackets moderate confidence signals.

${ }^{\mathrm{h}}$ Number of CCVs in the signal

' Multinomial regression summary statistics. Single variant analysis

j D: Distal regulation, P: proximal regulation, C: coding; na: prediction non available

k Predicted target genes with the largest confidence level for each variant. Between brackets, largest confidence level. na: prediction non available

' INQUISIT level of condifence

m Target genes with functional follow up 
Table 2. Regions in which target genes are predicted with high confidence

\begin{tabular}{|c|c|c|c|c|c|}
\hline Fine-mapping region $^{a}$ & $\begin{array}{l}\text { Signal / PP } \\
>30 \% \text { b }\end{array}$ & $\begin{array}{l}\mathbf{N} \\
\text { Variants }^{\mathrm{c}}\end{array}$ & Distal $^{d}$ & Promoter $^{\mathrm{e}}$ & Coding $^{f}$ \\
\hline chr1:10043820-11066215 & Signal 1 & 19 & PEX14 & & \\
\hline \multirow[t]{2}{*}{ chr1:18307339-19307339 } & Signal 1 & 12 & & $K L H D C 7 A$ & \\
\hline & $P P>30 \%$ & 1 & & $K L H D C 7 A$ & \\
\hline \multirow[t]{2}{*}{ chr1:46100917-47100917 } & Signal 1 & 11 & LRRC41, PIK3R3 & & \\
\hline & $P P>30 \%$ & 2 & MAST2, POMGNT1 & & \\
\hline chr1:113948389-114948389 & Signal 1 & 12 & $R S B N 1$ & & \\
\hline \multirow[t]{2}{*}{ chr1:145144984-146144984 } & Signal 1 & 35 & NUDT17, PDZK1, RNF115 & & \\
\hline & Signal 2 & 5 & PIAS3 & & \\
\hline chr1:154648781-155648781 & Signal 1 & 16 & $\begin{array}{l}\text { EFNA1, FAM189B, GBA, MTX1, MUC1, RP11- } \\
263 K 19.4, \text { SLC50A1, THBS3, TRIM46 }\end{array}$ & & \\
\hline \multirow[t]{2}{*}{ chr1:200937832-201937832 } & Signal 1 & 2 & TNNI1 & & \\
\hline & $\mathrm{PP}>30 \%$ & 2 & TNNI1 & & \\
\hline chr1:203301249-204301249 & Signal 1 & 56 & SOX13 & & \\
\hline chr1:204018842-205018842 & Signal 1 & 6 & LRRN2, MDM4, PIK3C2B, PPP1R15B & MDM4 & \\
\hline \multirow[t]{2}{*}{ chr1:241523898-242534263 } & Signal 1 & 2 & & & EXO1 \\
\hline & $\mathrm{PP}>30 \%$ & 2 & & & EXO1 \\
\hline chr2:18815791-19820803 & Signal 1 & 17 & OSR1 & & \\
\hline chr2:24687599-25687599 & Signal 1 & 92 & ADCY3, DNMT3A & & DNMT3A \\
\hline chr2:28670676-29670676 & Signal 1 & 81 & PPP1CB, TRMT61B & & \\
\hline \multirow[t]{3}{*}{ chr2:120745122-121746568 } & Signal 3 & 3 & $I N H B B$ & & \\
\hline & Signal 4 & 6 & $I N H B B$ & & \\
\hline & $\mathrm{PP}>30 \%$ & 3 & INHBB & & \\
\hline \multirow[t]{2}{*}{ chr2:171876221-173472971 } & Signal 2 & 35 & DYNC1/2 & & \\
\hline & $\mathrm{PP}>30 \%$ & 2 & DYNC1/2 & & \\
\hline \multirow[t]{2}{*}{ chr2:201681247-202681247 } & Signal 2 & 8 & CFLAR & & \\
\hline & CIMBA & 154 & CASP8, CFLAR, NIF3L1, PPIL3 & CASP8 & \\
\hline chr2:217405832-218796508 & $\mathrm{PP}>30 \%$ & 6 & IGFBP5 & & \\
\hline chr3:30175880-31175880 & Signal 1 & 23 & & & TGFBR2 \\
\hline chr3:46366866-47381488 & CIMBA & 16 & $C C D C 12, N B E A L 2$ & & \\
\hline chr3:63441697-64467900 & Signal 1 & 94 & ATXN7, PSMD6, PSMD6-AS2, THOC7 & & ATXN7 \\
\hline chr3:99223580-100223580 & Signal 1 & 92 & CMSS1, FILIP1L, TBC1D23 & & \\
\hline chr3:140612859-141612859 & Signal 1 & 24 & ZВТВ38 & & \\
\hline chr3:171785237-172785237 & CIMBA & 36 & TNFSF10 & & \\
\hline chr4:38312876-39312876 & Signal 1 & 24 & & & $T L R 1$ \\
\hline chr4:83870124-84870124 & Signal 1 & 84 & MRPS18C & MRPS18C & \\
\hline \multirow[t]{2}{*}{ chr4:105569013-106856761 } & Signal 1 & 21 & TET2 & & TET2 \\
\hline & $\mathrm{PP}>30 \%$ & 1 & AC004066.3, ARHGEF38 & & \\
\hline \multirow[t]{2}{*}{ chr5:779790-1797488 } & Signal 3 & 23 & CLPTM1L, TERT & & \\
\hline & $\mathrm{PP}>30 \%$ & 7 & CLPTM1L, TERT & & \\
\hline \multirow[t]{2}{*}{ chr5:55531884-56587883 } & Signal 1 & 3 & MAP3K1 & & \\
\hline & Signal 3 & 21 & MAP3K1 & & MAP3K1 \\
\hline
\end{tabular}




\begin{tabular}{|c|c|c|c|c|c|}
\hline & \\
\hline & Signal 4 & 70 & MAP3K1 & & \\
\hline & Signal 5 & 4 & MAP3К1 & & \\
\hline & $P P>30 \%$ & 5 & MAP3К1 & & \\
\hline chr5:90289470-91289470 & Signal 1 & 88 & ARRDC3 & $A R R D C 3$ & \\
\hline \multirow[t]{2}{*}{ chr5:131907058-132907058 } & Signal 1 & 117 & AFF4, HSPA4, ZCCHC10 & HSPA4 & \\
\hline & $\mathrm{PP}>30 \%$ & 1 & AFF4, HSPA4 & & \\
\hline chr5:157730013-158744083 & Signal 1 & 5 & EBF1 & & \\
\hline chr5:169091487-170091487 & Signal 1 & 19 & FOXI1 & & \\
\hline chr6:13213099-14222523 & Signal 1 & 51 & NOL7, RANBP9, RP1-223E5.4 & & \\
\hline chr6:20121238-21121238 & Signal 1 & 52 & CDKAL1 & & \\
\hline chr6:28422437-29426220 & Signal 1 & 36 & TRIM27 & & \\
\hline chr6:129849119-130849119 & Signal 1 & 43 & & & L3MBTL3 \\
\hline \multirow[t]{6}{*}{ chr6:151418856-152937016 } & Signal 1 & 1 & ESR1 & & \\
\hline & Signal 3 & 1 & ESR1 & & \\
\hline & Signal 4 & 6 & C6orf211, CCDC170, ESR1 & & \\
\hline & Signal 5 & 173 & & & ESR1 \\
\hline & Signal 6 & 22 & CCDC170, ESR1 & ESR1 & ESR1 \\
\hline & $\mathrm{PP}>30 \%$ & 4 & ESR1 & & \\
\hline chr7:91130620-92181597 & Signal 1 & 318 & KRIT1 & & AKAPG \\
\hline chr7:101054599-102054599 & Signal 1 & 6 & CUX1 & & CUX1 \\
\hline chr8:29009616-30009616 & Signal 1 & 16 & DUSP4 & & \\
\hline \multirow[t]{2}{*}{ chr8:101978959-102978959 } & Signal 1 & 47 & GRHL2, KB-1562D12.1, KB-1930G5.4 & & \\
\hline & $\mathrm{PP}>30 \%$ & 2 & KB-1930G5.4 & & \\
\hline chr8:116709548-117709548 & Signal 1 & 164 & TRPS1 & & \\
\hline \multirow[t]{3}{*}{ chr8:124110166-125110166 } & Signal 1 & 23 & FBXO32 & & \\
\hline & Signal 2 & 6 & FBXO32 & & \\
\hline & $\mathrm{PP}>30 \%$ & 1 & FBXO32, WDYHV1 & & \\
\hline \multirow[t]{2}{*}{ chr8:127424659-130041931 } & Signal 2 & 44 & MYC & & \\
\hline & $\mathrm{PP}>30 \%$ & 5 & MYC & & \\
\hline \multirow[t]{2}{*}{ chr9:109803808-111395353 } & Signal 4 & 1 & KLF4 & & \\
\hline & $\mathrm{PP}>30 \%$ & 6 & KLF4 & & \\
\hline \multirow[t]{2}{*}{ chr10:8588113-9588113 } & Signal 1 & 49 & GATA3 & & \\
\hline & $\mathrm{PP}>30 \%$ & 1 & GATA3 & & \\
\hline chr10:21532942-22909392 & Signal 2 & 58 & $B M I 1, C O M M D 3$ & & \\
\hline \multirow[t]{3}{*}{ chr10:80341148-81387721 } & Signal 1 & 10 & ZMIZ1 & & \\
\hline & Signal 3 & 9 & $\mathrm{ZCCHC24}$ & & \\
\hline & $\mathrm{PP}>30 \%$ & 2 & ZMIZ1 & & \\
\hline chr10:114273927-115286154 & Signal 2 & 42 & TCF7L2 & & \\
\hline \multirow[t]{6}{*}{ chr10:122593901-123849324 } & Signal 1 & 1 & FGFR2 & & \\
\hline & Signal 2 & 7 & FGFR2 & & \\
\hline & Signal 3 & 1 & & & FGFR2 \\
\hline & Signal 4 & 3 & & & FGFR2 \\
\hline & Signal 5 & 1 & FGFR2 & & \\
\hline & $P P>30 \%$ & 6 & FGFR2 & & FGFR2 \\
\hline
\end{tabular}




\begin{tabular}{|c|c|c|c|c|}
\hline chr11:303017-1303017 & Signal 1 & 19 & CD151, EPS8L2, PDDC1 & PIDD1 \\
\hline chr11:65043827-66083066 & Signal 1 & 13 & AP5B1, CFL1, OVOL1, RNASEH2C & \\
\hline \multirow[t]{4}{*}{ chr11:68831418-69879161 } & Signal 1 & 1 & CCND1, MYEOV & \\
\hline & Signal 2 & 3 & CCND1, MYEOV & \\
\hline & Signal 3 & 20 & CCND1, MYEOV & \\
\hline & $\mathrm{PP}>30 \%$ & 5 & CCND1, MYEOV & \\
\hline \multirow[t]{2}{*}{ chr11:107845515-108857137 } & Signal 1 & 59 & C11orf65, KDELC2 & ATM \\
\hline & CIMBA & 8 & & ATM \\
\hline chr12:13913931-14913931 & Signal 1 & 18 & ATF7IP & \\
\hline chr12:27639846-29034415 & Signal 2 & 375 & CCDC91, PTHLH & \\
\hline \multirow[t]{2}{*}{ chr12:95527759-96527759 } & Signal 1 & 2 & NTN4 & \\
\hline & $\mathrm{PP}>30 \%$ & 1 & NTN4 & \\
\hline \multirow[t]{4}{*}{ chr12:115336522-116336522 } & Signal 1 & 7 & $T B X 3$ & \\
\hline & Signal 2 & 1 & TBX3 & \\
\hline & Signal 3 & 8 & TBX3 & \\
\hline & $P P>30 \%$ & 7 & TBX3 & \\
\hline \multirow[t]{2}{*}{ chr12:120332146-121332146 } & Signal 1 & 5 & MSI1, RPLPO & \\
\hline & $\mathrm{PP}>30 \%$ & 1 & $R P L P O$ & \\
\hline chr13:32468810-33472626 & Signal 1 & 5 & & $B R C A 2$ \\
\hline \multirow[t]{2}{*}{ chr14:36632769-37635752 } & Signal 1 & 19 & PAX9, SLC25A21 & \\
\hline & $\mathrm{PP}>30 \%$ & 3 & PAX9 & \\
\hline \multirow[t]{3}{*}{ chr14:68117194-69534682 } & Signal 1 & 8 & ZFP36L1 & \\
\hline & Signal 2 & 4 & ZFP36L1 & \\
\hline & $\mathrm{PP}>30 \%$ & 3 & ZFP36L1 & \\
\hline chr14:92604072-93616351 & Signal 1 & 34 & & RIN3 \\
\hline chr15:91009215-92009215 & Signal 1 & 22 & $R C C D 1$ & \\
\hline chr16:3606788-4606788 & Signal 1 & 23 & ADCY9, CREBBP & \\
\hline \multirow[t]{2}{*}{ chr16:52038825-53038825 } & Signal 1 & 1 & TOX3 & \\
\hline & $\mathrm{PP}>30 \%$ & 2 & TOX3 & \\
\hline chr16:53300954-54355291 & Signal 1 & 6 & CTD-3032H12.2, FTO, IRX3 & \\
\hline chr16:54182064-55182064 & Signal 1 & 3 & CRNDE & \\
\hline \multirow[t]{2}{*}{ chr16:80148327-81150805 } & Signal 1 & 14 & $\begin{array}{l}\text { CDYL2, DYNLRB2, } \\
525 \text { RP11-18F14.4, }\end{array}$ & \\
\hline & $\mathrm{PP}>30 \%$ & 1 & DYNLRB2 & \\
\hline chr17:77281387-78281725 & Signal 1 & 10 & CCDC40 & \\
\hline \multirow[t]{2}{*}{ chr18:23832476-25075396 } & Signal 3 & 31 & KCTD1 & \\
\hline & $\mathrm{PP}>30 \%$ & 6 & KCTD1 & \\
\hline chr18:41899590-42899590 & Signal 1 & 38 & SETBP1 & SETBP 1 \\
\hline chr19:12658277-14454571 & Signal 1 & 21 & DAND5, GCDH, HOOK2, JUNB, MAST1, PRDX2 & \\
\hline \multirow[t]{2}{*}{ chr19:16795023-17894124 } & Signal 1 & 16 & $A B H D 8, M R P L 34$ & \\
\hline & CIMBA & 10 & ABHD8, MRPL34 & ANKLE1 \\
\hline chr19:18050434-19071141 & Signal 1 & 56 & $\begin{array}{l}\text { CRLF1, ELL, FKBP8, GDF15, ISYNA1, KXD1, } \\
\text { UBA52 }\end{array}$ & \\
\hline chr19:19048246-20048246 & Signal 1 & 162 & $\begin{array}{l}\text { ATP13A1, GATAD2A, LPAR2, MAU2, MEF2B, } \\
\text { MEF2BNB, NDUFA13, PBX4, RFXANK, SUGP1 }\end{array}$ & \\
\hline chr19:29777729-30777729 & Signal 1 & 60 & CCNE1 & \\
\hline
\end{tabular}




\begin{tabular}{|c|c|c|c|c|c|}
\hline chr19:43783447-44786513 & Signal 1 & 10 & KCNN4, PLAUR, SMG9 & & \\
\hline \multirow[t]{2}{*}{ chr21:16073983-17073983 } & Signal 1 & 7 & NRIP1 & & \\
\hline & $\mathrm{PP}>30 \%$ & 5 & NRIP1 & & \\
\hline \multirow[t]{4}{*}{ chr22:28000000-30121477 } & Signal 1 & 3 & & & CHEK2 \\
\hline & Signal 2 & 2 & & & CHEK2 \\
\hline & Signal 3 & 59 & CHEK2, CTA-292E10.6, EWSR1, XBP1 & CHEK2 & CHEK2 \\
\hline & $\mathrm{PP}>30 \%$ & 3 & CHEK2, XBP1 & & \\
\hline \multirow[t]{2}{*}{ chr22:38068833-39859355 } & Signal 1 & 27 & MAFF, PLA2G6, TMEM184B & & \\
\hline & Signal 3 & 4 & CBX6, NPTXR, SUN2 & & \\
\hline chr22:40376234-41527870 & Signal 1 & 196 & MKL1, SLC25A17 & & \\
\hline chr22:41538786-42538786 & Signal 1 & 10 & & & EP300 \\
\hline chr22:45783297-46783297 & Signal 1 & 34 & WNT7B & & \\
\hline
\end{tabular}

\section{a GRCh37/hg19, bp}

${ }^{\mathrm{b}}$ Independent signal or set of variants with $\mathrm{PP} \geq 30 \%$

${ }^{c}$ Number of $\mathrm{CCV}$ or number of variants with $\mathrm{PP} \geq 30 \%$

High confidence target genes by ${ }^{d}$ distal regulatory, ${ }^{e}$ promoter, or ${ }^{f}$ coding variants at the signal or at the set of variants with $\mathrm{PP} \geq 30 \%$ 